\title{
LOCALIZING VIRTUAL STRUCTURE SHEAVES BY COSECTIONS
}

\author{
YOUNG-HOON KIEM AND JUN LI
}

\begin{abstract}
We construct a cosection localized virtual structure sheaf when a Deligne-Mumford stack is equipped with a perfect obstruction theory and a cosection of the obstruction sheaf.
\end{abstract}

\section{IntRoduction}

According to Schubert 25], enumerative geometry is about finding the number of geometric figures of fixed type satisfying certain given conditions. A typical way of solving an enumerative problem consists of constructing a moduli space parameterizing all geometric figures of fixed type and then finding the number of intersection points of the subsets defined by the given conditions. The latter part is called an intersection theory and a solid mathematical theory was developed by Fulton and MacPherson [13] in 1970s through a systematic use of normal cones and Gysin maps. In particular, the intersection rings for smooth schemes were rigorously defined and Riemann-Roch theorems were established for schemes. Fulton's intersection theory was updated for Deligne-Mumford stacks by Vistoli [37] and for Artin stacks by Kresch [27.

However moduli spaces are often very singular and do not behave well under deformation. To deal with this issue, the theory of virtual fundamental classes was developed in 1990s by Li-Tian [30] and Behrend-Fantechi [2]. A Deligne-Mumford stack $X$ has its intrinsic normal cone $\mathfrak{C}_{X}$ which is locally defined as the quotient stack $C_{U / M} /\left.T_{M}\right|_{U}$ for an étale $U \rightarrow X$ and a closed embedding $U \hookrightarrow M$ into a smooth $M$. When $\mathfrak{C}_{X}$ is embedded into a vector bundle stack $\mathcal{E}_{X}$ which is locally the quotient $E_{1} / E_{0}$ for vector bundles $E_{1}, E_{0}$, the virtual fundamental class is defined as the intersection

$$
[X]^{\text {vir }}=0_{\mathcal{E}_{X}}^{!}\left[\mathfrak{C}_{X}\right] \in A_{*}(X)
$$

of $\mathfrak{C}_{X}$ with the zero section of $\mathcal{E}_{X}$. Many nice properties such as deformation invariance can be deduced under reasonable assumptions [24] and since 1995, important enumerative invariants in algebraic geometry have been constructed as integrals on the virtual cycles $[X]^{\text {vir }}$ on suitable moduli spaces $X$,

Date: 2018.9.1.

YHK was partially supported by Samsung Science and Technology Foundation grant SSTF-BA1601-01; JL was partially supported by National Science Foundation, DMS1601211. 
including the Gromov-Witten, Donaldson-Thomas, Pandharipande-Thomas invariants and more.

The computation of these virtual invariants is known to be very hard because there are not many tools to handle the virtual cycle $[X]^{\text {vir }}$. When there is an action of $\mathbb{C}^{*}$ on $X$, under suitable assumptions, the virtual cycle is localized to the fixed locus $X^{\mathbb{C}^{*}}$ by the torus localization formula (cf. [16])

$$
[X]^{\text {vir }}=\frac{\left[X^{\mathbb{C}^{*}}\right]^{\text {vir }}}{e\left(N^{\text {vir }}\right)}
$$

which has been most effective for actual computations so far. Recently, another localization of the virtual cycle $[X]^{\text {vir }}$ was discovered [19] which also turned out to be quite useful [4, 6, 7, 8, 10, 11, 14, 15, 17, 18, 21, 22, 26, 32, 33 . It says that when there is a morphism $\sigma: \mathcal{E}_{X} \rightarrow \mathcal{O}_{X}$, called a cosection, the virtual cycle is localized to the zero locus $X(\sigma)$ of $\sigma$, i.e. we have a class $[X]_{\text {loc }}^{\text {vir }} \in A_{*}(X(\sigma))$ which equals $[X]^{\text {vir }}$ when pushed forward to $X$. The two localizations were combined in [5].

Recently there arose a demand to lift the theory of virtual cycles in Chow groups to algebraic K-groups with applications towards physics and geometric representation theory. In 2, 29, the K-theoretic virtual fundamental class was defined as

$$
\left[\mathcal{O}_{X}^{\mathrm{vir}}\right]=0_{E_{1}}^{!}\left[\mathcal{O}_{C_{1}}\right]=\left[\mathcal{O}_{X} \otimes_{\mathcal{O}_{E_{1}}}^{L} \mathcal{O}_{C_{1}}\right] \in K_{0}(X)
$$

and called the virtual structure sheaf of $X$. Here $C_{1}=\mathfrak{C}_{X} \times \mathcal{E}_{X} E_{1}$ when $\mathcal{E}_{X}=E_{1} / E_{0}$ is a global resolution by vector bundles. In order to lift some of the results on the virtual cycles and invariants to the setting of algebraic Kgroups, it is necessary to develop K-theoretic techniques to handle the virtual structure sheaf $\left[\mathcal{O}_{X}^{\text {vir }}\right]$ such as the cosection localization. In this paper, we prove the following.

Theorem 1.1. (Theorem 5.1, Proposition 5.2, Proposition 5.5) Let $X$ be a Deligne-Mumford stack equipped with a perfect obstruction theory $\phi: E \rightarrow$ $\mathbb{L}_{X}$ and a cosection $\sigma: h^{1}\left(E^{\vee}\right) \rightarrow \mathcal{O}_{X}$ whose zero locus is denoted by $X(\sigma)$. Assume $E$ admits a global resolution $\left[E^{-1} \rightarrow E^{0}\right]$ by locally free sheaves (e.g. if $X$ is quasi-projective). Then there is a cosection localized virtual structure sheaf

$$
\left[\mathcal{O}_{X, \mathrm{loc}}^{\mathrm{vir}}\right] \in K_{0}(X(\sigma))
$$

such that $\imath_{*}\left[\mathcal{O}_{X, \text { loc }}^{\mathrm{vir}}\right]=\left[\mathcal{O}_{X}^{\mathrm{vir}}\right] \in K_{0}(X)$ where $\imath: X(\sigma) \rightarrow X$ denotes the inclusion. Moreover, $\left[\mathcal{O}_{X, \mathrm{loc}}^{\mathrm{vir}}\right]$ is independent of the choice of the resolution $\left[E^{-1} \rightarrow E^{0}\right]$ and is deformation invariant.

By [19, §4], the cone $C_{1} \subset E_{1}$ has (reduced) support in

$$
E_{1}(\sigma)=\left.E_{1}\right|_{X(\sigma)} \cup \operatorname{ker}\left(\sigma:\left.E_{1}\right|_{U} \rightarrow \mathcal{O}_{U}\right)
$$

where $U=X-X(\sigma)$. We define the cosection localized Gysin map (cf. Theorem 4.1)

$$
0_{E_{1}, \sigma}^{!}: K_{0}\left(E_{1}(\sigma)\right) \rightarrow K_{0}(X(\sigma))
$$


and the cosection localized virtual structure sheaf $\left[\mathcal{O}_{X, \text { loc }}^{\mathrm{vir}}\right]$ is defined as

$$
\left[\mathcal{O}_{X, \text { loc }}^{\operatorname{vir}}\right]=0 !_{E_{1}, \sigma}\left[\mathcal{O}_{C_{1}}\right] \in K_{0}(X(\sigma)) .
$$

Using the cosection localized virtual structure sheaf $\left[\mathcal{O}_{X, \text { loc }}^{\text {vir }}\right] \in K_{0}(X(\sigma))$, we can define the cosection localized virtual Euler characteristic, even when $X$ is not proper, as long as $X(\sigma)$ is proper.

Definition 1.2. The cosection localized virtual Euler characteristic of a class $\beta \in K^{0}(X)$ is defined as

$$
\chi_{\mathrm{loc}}^{\mathrm{vir}}(X, \beta)=\chi\left(X(\sigma), \beta \cdot \mathcal{O}_{X, \text { loc }}^{\mathrm{vir}}\right)=\sum_{i}(-1)^{i} \operatorname{dim} H^{i}\left(X(\sigma), \beta \cdot \mathcal{O}_{X, \text { loc }}^{\mathrm{vir}}\right) .
$$

As an application, we lift the results of [6, 7] to the K-theoretic setting. In particular, we define the K-theoretic Fan-Jarvis-Ruan-Witten invariant for narrow sector (which doesn't involve the matrix factorization machinery of Polishchuk and Vaintrob) as the Euler characteristic of the virtual structure sheaf on the moduli space of spin curves with sections.

The layout of this paper is as follows. In $\S 2$, we collect useful facts. In $\S 3$, we prove that the virtual structure sheaf vanishes if there is a surjective cosection $\sigma: O b_{X} \rightarrow \mathcal{O}_{X}$. In $\S 4$, we construct the cosection localized Gysin map $0 ! E_{1}, \sigma$. In $\S 5$, we define the cosection localized virtual structure sheaf $\left[\mathcal{O}_{X, \text { loc }}^{\mathrm{vir}}\right]$ and prove key properties. In $\S 6$, we apply the cosection localized virtual structure sheaf to construct a K-theoretic FJRW invariant and discuss the K-theoretic Landau-Ginzburg/Calabi-Yau correspondence.

In this paper, all schemes or Deligne-Mumford stacks are separated and Noetherian of finite type over the complex number field $\mathbb{C}$. When $X \hookrightarrow Y$ is a closed embedding, its normal cone is denoted by $C_{X / Y}$. When $f: X \rightarrow Y$ is a morphism and $F$ is a coherent sheaf on $Y,\left.F\right|_{Y}$ denotes the (underived) pullback $f^{*} F$.

We thank Richard Thomas for useful comments including Remark 3.3.

\section{Preliminaries}

In this section, we collect useful facts about algebraic K-groups and cosection localization.

2.1. Algebraic K-theory. For a Deligne-Mumford stack $X, K_{0}(X)$ (resp. $K^{0}(X)$ ) denotes the Grothendieck group generated by coherent sheaves (resp. locally free sheaves) on $X$ with relations $[F]=\left[F^{\prime}\right]+\left[F^{\prime \prime}\right]$ whenever we have an exact sequence $0 \rightarrow F^{\prime} \rightarrow F \rightarrow F^{\prime \prime} \rightarrow 0$.

For a projective morphism $f: X \rightarrow Y$, the pushforward

$$
f_{*}: K_{0}(X) \longrightarrow K_{0}(Y)
$$


is defined by the right derived direct image $R f_{*}$ as

$$
f_{*}[F]=\left[R f_{*} F\right]=\sum_{i}(-1)^{i}\left[R^{i} f_{*} F\right] .
$$

The pullback

$$
f^{*}: K_{0}(Y) \longrightarrow K_{0}(X)
$$

for a morphism $f: X \rightarrow Y$ is defined by the left derived inverse image $L f^{*}$ as

$$
f^{*}[G]=\left[L f^{*} G\right]=\left[\mathcal{O}_{X} \otimes_{f^{-1} \mathcal{O}_{Y}}^{L} f^{-1} G\right]=\sum_{i}(-1)^{i}\left[\operatorname{Tor}_{i}^{f^{-1} \mathcal{O}_{Y}}\left(\mathcal{O}_{X}, f^{-1} G\right)\right]
$$

when the sum is finite, i.e. $\operatorname{Tor}_{i}^{f^{-1} \mathcal{O}_{Y}}\left(\mathcal{O}_{X}, f^{-1} G\right) \neq 0$ for only finitely many $i$. Tensor product of locally free sheaves makes $K^{0}(X)$ a commutive ring and $K_{0}(X)$ a module over $K^{0}(X)$.

For a Deligne-Mumford stack $X$, let $X_{\text {red }}$ be the reduced stack of $X$ and $\imath: X_{\text {red }} \rightarrow X$ denote the inclusion.

Proposition 2.1. For a Deligne-Mumford stack $X, \imath_{*}: K_{0}\left(X_{\text {red }}\right) \rightarrow K_{0}(X)$ is an isomorphism.

Proof. Let $I$ be the ideal sheaf defining $X_{\text {red }}$ in $X$, i.e. it is the ideal of nilpotents. Since $X$ is Noetherian by assumption, $I^{n}=0$ for some $n>0$. Hence, if $F$ is a coherent sheaf on $X$,

$$
[F]=\sum_{r=0}^{n-1}\left[I^{r} F / I^{r+1} F\right] \in K_{0}(X)
$$

and $I^{r} F / I^{r+1} F$ are coherent sheaves on $X_{\text {red }}$. Hence $\imath_{*}$ is surjective. In fact, the assignment

$$
[F] \mapsto \sum_{r=0}^{n-1}\left[I^{r} F / I^{r+1} F\right]
$$

is obviously a right inverse of $\imath_{*}$ and hence $\imath_{*}$ is injective. It is a straightforward exercise to check that (2.1) is a homomorphism of $K$-groups.

Proposition 2.2. [3, Lemma 17] For a Deligne-Mumford stack $X, K_{0}(X)$ is generated by the classes $\left[\mathrm{O}_{Z}\right]$ for integral closed substacks $Z$ in $X$.

Proof. By Proposition 2.1, we may assume $X$ is reduced and consider only sheaves supported on reduced stacks. Now the proof of [3, Lemma 17], by induction on the dimension of the support, also proves the proposition.

Proposition 2.3. 3, Proposition 7] Let $X$ be a Deligne-Mumford stack and $Z$ be a closed substack. Let $U=X-Z$. Then the inclusions

$$
Z \stackrel{\imath}{\longleftrightarrow} X \stackrel{\jmath}{\longleftarrow} U
$$

induce a complex

$$
K_{0}(Z) \stackrel{\imath_{*}}{\longrightarrow} K_{0}(X) \stackrel{\jmath^{*}}{\longrightarrow} K_{0}(U) \longrightarrow 0
$$


which is exact at $K_{0}(U)$. If every coherent sheaf on $U$ extends to a coherent sheaf on $X$ (e.g. $X$ is a scheme), then (2.2) is exact.

Proof. $\jmath^{*}$ is surjective by Proposition 2.2 since $\jmath^{*}\left[\mathcal{O}_{\bar{Z}}\right]=\left[\mathcal{O}_{Z}\right]$ for an integral $Z \subset U$ where $\bar{Z}$ denotes the closure of $Z$ in $X$. When every coherent sheaf on $U$ extends to a coherent sheaf on $X$, the exactness of (2.2) is proved by the same proof of [3, Proposition 7].

For instance, when $X$ has the resolution property, every sheaf of $\mathcal{O}_{U^{-}}$ modules, where $U \subset X$ open, can be extended to a sheaf of $\mathcal{O}_{X}$-modules. When the Keel-Mori coarse moduli of $X$ is a separated scheme, $X$ has the resolution property [36].

2.2. Virtual structure sheaf. Let $X$ be a Deligne-Mumford stack equipped with a perfect obstruction theory $\phi: E \rightarrow \mathbb{L}_{X}$, i.e. $\phi$ is a morphism in the derived category of quasi-coherent sheaves on $X$ such that $h^{0}(\phi): h^{0}(E) \rightarrow$ $h^{0}\left(\mathbb{L}_{X}\right)$ is an isomorphism, $h^{-1}(\phi): h^{-1}(E) \rightarrow h^{-1}\left(\mathbb{L}_{X}\right)$ is surjective, and $E$ is locally isomorphic to a 2-term complex of locally free sheaves, concentrated in degrees -1 and 0 . Here $\mathbb{L}_{X}=\tau^{\geq-1} L_{X}$ is the truncated cotangent complex of $X$.

To avoid discussion on algebraic K-theory of Artin stacks, let us suppose in this paper that $E$ admits a global resolution by a 2-term complex $\left[E^{-1} \rightarrow\right.$ $\left.E^{0}\right]$ of locally free sheaves on $X$. Let $\left[E_{0} \rightarrow E_{1}\right]$ denote the dual with $E_{i}=\left(E^{-i}\right)^{\vee}$. By [2], the intrinsic normal cone $\mathfrak{C}_{X}$ of $X$ is locally the Artin stack $C_{X / M} /\left.T_{M}\right|_{X}$ if $X \rightarrow M$ is a closed embedding into a smooth DeligneMumford stack. Here $C_{X / M}$ denotes the normal cone of $X$ in $M$. Then $h^{1} / h^{0}\left(\phi^{\vee}\right)$ embeds $\mathfrak{C}_{X}$ into $\mathcal{E}_{X}=E_{1} / E_{0}$. The intrinsic normal cone $\mathfrak{C}_{X}$ lifts to a cone

$$
C_{1}:=\mathfrak{C}_{X} \times \mathcal{E}_{X} E_{1} \subset E_{1}
$$

and the virtual fundamental class is defined as

$$
[X]^{\text {vir }}=0 !_{\mathcal{E}_{X}}^{!}\left[\mathfrak{C}_{X}\right]=0 ! E_{E_{1}}\left[C_{1}\right]
$$

Likewise, the virtual structure sheaf of $X$ is defined as the sheaf theoretic intersection

$$
\left[\mathcal{O}_{X}^{\mathrm{vir}}\right]=\left[\mathcal{O}_{X} \otimes_{\mathcal{O}_{E_{1}}}^{L} \mathcal{O}_{C_{1}}\right]=\sum_{i}(-1)^{i}\left[\operatorname{Tor}_{i}^{E_{1}}\left(\mathcal{O}_{X}, \mathcal{O}_{C_{1}}\right)\right] \in K_{0}(X)
$$

where $\otimes_{\mathcal{O}_{E_{1}}}^{L}$ is the derived tensor product (cf. [2, Remark 5.4]). If we denote the bundle projection $E_{1} \rightarrow X$ by $\pi$, the tautological section of $\pi^{*} E_{1}$ over $E_{1}$ gives us the Koszul resolution $\wedge \pi^{*} E_{1}^{\vee}$ of the sheaf $\mathcal{O}_{X}$ on $E_{1}$. Hence (2.3) can be rephrased as

$$
\left[\mathcal{O}_{X}^{\mathrm{vir}}\right]=\left[\wedge^{*} \pi^{*} E_{1}^{\vee} \otimes_{\mathcal{O}_{E_{1}}} \mathcal{O}_{C_{1}}\right]
$$


2.3. Localizing virtual fundamental classes by cosections. Suppose the obstruction sheaf $O b_{X}:=h^{1}\left(E^{\vee}\right)$ admits a cosection $\sigma: O b_{X} \rightarrow \mathcal{O}_{X}$. Let $X(\sigma)$ denote the locus where $\sigma$ is not surjective, i.e. the closed stubstack defined by the ideal sheaf $\sigma\left(O b_{X}\right) \subset \mathcal{O}_{X}$. By [19, §4], the (reduced) support of $C_{1}$ is contained in

$$
E_{1}(\sigma)=\left.E_{1}\right|_{X(\sigma)} \cup \operatorname{ker}\left(\sigma: E_{1} \rightarrow O b_{X} \rightarrow \mathcal{O}_{X}\right) .
$$

The ordinary Gysin map $0 !_{E_{1}}: A_{*}\left(E_{1}\right) \rightarrow A_{*}(X)$ can be localized to a homomorphism (cf. [19, §2])

$$
0 ! E_{E_{1}, \sigma}: A_{*}\left(E_{1}(\sigma)\right) \longrightarrow A_{*}(X(\sigma))
$$

such that $\imath_{*} \circ 0_{E_{1}, \sigma}^{!}=0_{E_{1}}^{!}$where $\imath: X(\sigma) \rightarrow X$ is the inclusion map.

The cosection localized virtual cycle is then obtained by

$$
[X]_{\mathrm{loc}}^{\mathrm{vir}}=0_{E_{1}, \sigma}^{!}\left[C_{1}\right] \in A_{*}(X(\sigma))
$$

which satisfies many nice properties such as $\imath_{*}[X]_{\text {loc }}^{\text {vir }}=[X]^{\text {vir }}$ and deformation invariance. When $X$ is not proper, $[X]^{\text {vir }}$ is not properly supported in general. If $X(\sigma)$ is proper, then we can still define integrals on the cosection localized virtual fundamental class $[X]_{\text {loc }}^{\mathrm{vir}}$.

In the subsequent sections, we will show that the virtual structure sheaf $\left[\mathcal{O}_{X}^{\mathrm{vir}}\right] \in K_{0}(X)$ is also localized to $X(\sigma)$ by the cosection.

\section{A VAnishing Result For Virtual struCture sheaves}

In this section, we show that if there is a surjective cosection $\sigma: O b_{U} \rightarrow$ $\mathcal{O}_{U}$ on a Deligne-Mumford stack $U$, then the virtual structure sheaf $\left[\mathcal{O}_{U}^{\mathrm{vir}}\right] \in$ $K_{0}(U)$ is zero.

Let $U$ be a Deligne-Mumford stack and let $\phi: E_{U} \rightarrow \mathbb{L}_{U}$ be a perfect obstruction theory on $U$ where $\mathbb{L}_{U}=\tau^{\geq-1} L_{U}$ is the truncated cotangent complex on $U$, i.e. $h^{0}(\phi)$ is an isomorphism and $h^{-1}(\phi)$ is surjective. By [2], we have a closed embedding

$$
\mathfrak{N}_{U}=h^{1} / h^{0}\left(\mathbb{L}_{U}^{\vee}\right) \hookrightarrow h^{1} / h^{0}\left(E_{U}^{\vee}\right)=: \mathcal{E}_{U}
$$

of the virtual normal sheaf $\mathfrak{N}_{U}$ into the vector bundle stack $\mathcal{E}_{U}$. In this section, we make following assumptions.

Assumption 3.1. (1) There is a surjective homomorphism $\sigma_{U}: O b_{U} \rightarrow \mathcal{O}_{U}$ where $O b_{U}=h^{1}\left(E_{U}^{\vee}\right)$ is the obstruction sheaf.

(2) There is a 2-term complex $\left[E^{-1} \rightarrow E^{0}\right]$ of locally free sheaves on $U$ which is isomorphic to $E_{U}$ in the derived category $D\left(\mathcal{O}_{U}\right)$.

Let $E_{i}=\left(E^{-i}\right)^{\vee}$ be the dual bundle of $E^{-i}$ for $i=0,1$ so that $E_{U}^{\vee} \cong$ $\left[E_{0} \rightarrow E_{1}\right]$. Then $\mathcal{E}_{U}=h^{1} / h^{0}\left(E_{U}^{\vee}\right)$ is the quotient stack $\left[E_{1} / E_{0}\right]$. Since the abelian hull of the intrinsic normal cone $\mathfrak{C}_{U}$ is the intrinsic normal sheaf $\mathfrak{N}_{U}$, we have an embedding

$$
\mathfrak{C}_{U} \hookrightarrow \mathfrak{N}_{U} \hookrightarrow \mathcal{E}_{U}
$$


uniquely determined by the perfect obstruction theory $\phi$. The natural homomorphism $E_{1} \rightarrow \mathcal{E}_{U}=\left[E_{1} / E_{0}\right]$ induces the Cartesian square

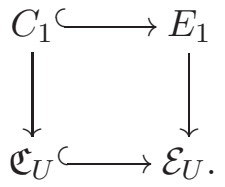

Since $O b_{U}$ is the cokernel of $E^{\vee}=\left[E_{0} \rightarrow E_{1}\right]$, we have a surjective homomorphism

$$
E_{1} \longrightarrow O b_{U} \stackrel{\sigma_{U}}{\longrightarrow} \mathcal{O}_{U}
$$

By [19, §4], $C_{1}$ has support in the subbundle

$$
E_{1}^{\prime}=\operatorname{ker}\left(E_{1} \rightarrow \mathcal{O}_{U}\right) .
$$

By Proposition 2.1, we may think of $\mathcal{O}_{C_{1}}$ as a sheaf on $E_{1}^{\prime}$ because

$$
\left[\mathcal{O}_{C_{1}}\right] \in K_{0}\left(E_{1}^{\prime}\right) \text {. }
$$

For the computation of $\mathcal{O}_{U}^{\mathrm{vir}}$, we may use the Koszul resolution of $\mathcal{O}_{U}$ as an $\mathcal{O}_{E_{1}}$-module. If we denote the vector bundle projection of $E_{1}$ by $\pi: E_{1} \rightarrow U$, the tautological section $\mathcal{O}_{E_{1}} \rightarrow \pi^{*} E_{1}$ induces a complex

$$
0 \longrightarrow \wedge^{r} \pi^{*} E_{1}^{\vee} \longrightarrow \cdots \longrightarrow \wedge^{2} \pi^{*} E_{1}^{\vee} \longrightarrow \pi^{*} E_{1}^{\vee} \longrightarrow \mathcal{O}_{E_{1}} \longrightarrow 0
$$

where $r=\operatorname{rank}\left(E_{1}\right)$. As is well known, this complex is a locally free resolution of $\mathcal{O}_{U}$ on $E_{1}$, and $\operatorname{Tor}_{i}^{E_{1}}\left(\mathcal{O}_{C_{1}}, \mathcal{O}_{U}\right)$ is the $(-i)$-th cohomology of the complex

$$
0 \rightarrow \wedge^{r} \pi^{*} E_{1}^{\vee} \otimes_{\mathcal{O}_{E_{1}}} \mathcal{O}_{C_{1}} \rightarrow \cdots \rightarrow \wedge^{1} \pi^{*} E_{1}^{\vee} \otimes_{\mathcal{O}_{E_{1}}} \mathcal{O}_{C_{1}} \rightarrow \mathcal{O}_{C_{1}} \rightarrow 0 .
$$

In this section, we prove the following.

Proposition 3.2. Let $\sigma_{U}: O b_{U} \rightarrow \mathcal{O}_{U}$ be a surjective homomorphism. Then the virtual structure sheaf $\left[\mathcal{O}_{U}^{\mathrm{vir}}\right] \in K_{0}(U)$ is zero.

Proof. Let $E_{1}^{\prime}$ be the kernel of the surjective homomorphism (3.1) so that we have an exact sequence of vector bundles

$$
0 \longrightarrow E_{1}^{\prime} \longrightarrow E_{1} \longrightarrow \mathcal{O}_{U} \longrightarrow 0
$$

which induces an exact sequence of vector bundles

$$
0 \longrightarrow \wedge^{i-1} E_{1}^{\prime \vee} \longrightarrow \wedge^{i} E_{1}^{\vee} \longrightarrow \wedge^{i} E_{1}^{\prime \vee} \longrightarrow 0
$$

for each $i$, where the superscript $\vee$ denotes the dual vector bundle. Recall that the normal cone $C_{1}$ has support in $E_{1}^{\prime}$ by [19. Pulling back (3.3) by $\pi$ and tensoring with $\mathcal{O}_{C_{1}}$ give us a short exact sequence

$0 \longrightarrow \wedge^{\cdot-1} \pi^{*} E_{1}^{\prime \vee} \otimes_{\mathcal{O}_{E_{1}}} \mathcal{O}_{C_{1}} \longrightarrow \wedge^{*} \pi^{*} E_{1}^{\vee} \otimes_{\mathcal{O}_{E_{1}}} \mathcal{O}_{C_{1}} \longrightarrow \wedge^{*} \pi^{*} E_{1}^{\prime \vee} \otimes_{\mathcal{O}_{E_{1}}} \mathcal{O}_{C_{1}} \longrightarrow 0$

of complexes of coherent sheaves on $C_{1} \subset E_{1}^{\prime}$. Then we have

$$
\wedge \pi^{*} E_{1}^{\prime \vee} \otimes_{\mathcal{O}_{E_{1}}} \mathcal{O}_{C_{1}} \cong\left(\wedge^{*} \pi^{*} E_{1}^{\prime \vee} \otimes_{\mathcal{O}_{E_{1}}} \mathcal{O}_{E_{1}^{\prime}}\right) \otimes_{\mathcal{O}_{E_{1}^{\prime}}} \mathcal{O}_{C_{1}}=\wedge^{\cdot} \pi^{\prime *} E_{1}^{\prime \vee} \otimes_{\mathcal{O}_{E_{1}^{\prime}}} \mathcal{O}_{C_{1}}
$$


where $\pi^{\prime}: E_{1}^{\prime} \rightarrow U$ is the bundle projection. Let

$$
A_{j}=\operatorname{Tor}_{j}^{E_{1}}\left(\mathcal{O}_{U}, \mathcal{O}_{C_{1}}\right) \quad \text { and } \quad A_{j}^{\prime}=\operatorname{Tor}_{j}^{E_{1}^{\prime}}\left(\mathcal{O}_{U}, \mathcal{O}_{C_{1}}\right)
$$

so that

$$
\mathcal{O}_{U}^{\mathrm{vir}}=\sum_{j}(-1)^{j} A_{j}
$$

The long exact sequence associated to (3.4) gives us the exact sequence

$$
0 \longrightarrow A_{r} \longrightarrow A_{r}^{\prime} \longrightarrow A_{r-1}^{\prime} \longrightarrow \cdots \longrightarrow A_{0}^{\prime} \longrightarrow A_{1} \longrightarrow A_{1}^{\prime} \longrightarrow 0
$$

and an isomorphism $A_{0}=A_{0}^{\prime}$ which imply

$$
\sum_{j}(-1)^{j} A_{j}=\sum_{j}(-1)^{j} A_{j-1}^{\prime}+\sum_{j}(-1)^{j} A_{j}^{\prime}=0 .
$$

Therefore we have the vanishing

$$
\left[\mathcal{O}_{U}^{\text {vir }}\right]=\sum_{j}(-1)^{j} A_{j}=0 \in K_{0}(U) .
$$

This proves the proposition.

Remark 3.3. Richard Thomas kindly informed us of a simple proof of Proposition 3.2 as follows. The normal bundle $N$ of $E_{1}^{\prime}$ in $E_{1}$ is the trivial line bundle. Letting $\imath: E^{\prime} \rightarrow E$ denote the inclusion, for a coherent sheaf $\alpha$ on $E_{1}^{\prime}$ like $\mathcal{O}_{C_{1}}$, the exact triangle

$$
N^{\vee} \otimes \alpha[-1] \rightarrow L \imath^{*} \imath_{*} \alpha \rightarrow \alpha \rightarrow N^{\vee} \otimes \alpha
$$

shows that $\left[L \imath^{*} \imath_{*} \alpha\right]=\left[\mathcal{O}_{E_{1}^{\prime}} \otimes_{\mathcal{O}_{E_{1}}}^{L} \alpha\right]=0 \in K_{0}\left(E_{1}^{\prime}\right)$. This certainly suffices for the vanishing $\left[\mathcal{O}_{U}^{\text {vir }}\right]=\left[\mathcal{O}_{U} \otimes_{\mathcal{O}_{E_{1}}}^{L} \mathcal{O}_{C_{1}}\right]=0$.

In many natural situations, the cosection $\sigma$ is not surjective. Let $X(\sigma)$ be the zero locus of a cosection $\sigma: O b_{X} \rightarrow \mathcal{O}_{X}$ of the obstruction sheaf $O b_{X}$ on a Deligne-Mumford stack $X$. Then $\sigma$ is surjective on $U=X-X(\sigma)$ and hence we have the vanishing

$$
\jmath^{*}\left[\mathcal{O}_{X}^{\mathrm{vir}}\right]=\left[\mathcal{O}_{U}^{\mathrm{vir}}\right]=0 \in K_{0}(U)
$$

where $\jmath: U \rightarrow X$ denotes the inclusion. By (2.2), when $X$ has the resolution property, we should have

$$
\imath_{*}(\xi)=\left[\mathcal{O}_{X}^{\mathrm{vir}}\right], \quad \exists \xi \in K_{0}(X(\sigma))
$$

where $\imath: X(\sigma) \rightarrow X$ is the inclusion. In the subsequent sections, we will show that the virtual structure sheaf $\left[\mathcal{O}_{X}^{\mathrm{vir}}\right]$ localizes to the substack $X(\sigma)$ in a canonical manner. 


\section{Cosection localized Gysin map}

Let $X$ be a Deligne-Mumford stack and $E_{1}$ be a vector bundle on $X$. Let

$$
\sigma: E_{1} \longrightarrow \mathcal{O}_{X}
$$

be a homomorphism of $\mathcal{O}_{X}$-modules and $X(\sigma)$ be the zero locus of $\sigma$, i.e. the closed substack defined by the ideal sheaf $\sigma\left(E_{1}\right) \subset \mathcal{O}_{X}$.

Let $U=X-X(\sigma)$ and let

$$
E_{1}(\sigma):=\left.E_{1}\right|_{X(\sigma)} \cup \operatorname{ker}\left(\sigma:\left.E_{1}\right|_{U} \rightarrow \mathcal{O}_{U}\right) .
$$

Here we are now considering $E_{1}$ to be the total space of the vector bundle $E_{1}$ and likewise $E_{1}(\sigma)$ is a substack of $E_{1}$.

The purpose of this section is to construct the following cosection localized Gysin map.

Theorem 4.1. Under the notation as above, we have a homomorphism

$$
0_{E_{1}, \sigma}^{!}: K_{0}\left(E_{1}(\sigma)\right) \longrightarrow K_{0}(X(\sigma))
$$

which satisfies

$$
\imath_{*} \circ 0_{E_{1}, \sigma}^{!}=0_{E_{1}}^{!} \circ \jmath_{*}: K_{0}\left(E_{1}(\sigma)\right) \longrightarrow K_{0}(X)
$$

where $\imath: X(\sigma) \rightarrow X$ and $\jmath: E_{1}(\sigma) \rightarrow E_{1}$ denote the inclusion maps while

$$
0_{E_{1}}^{!}[F]=\left[\mathcal{O}_{X} \otimes_{\mathcal{O}_{E_{1}}}^{L} F\right]=\sum_{i}(-1)^{i} \operatorname{Tor}_{i}^{E_{1}}\left(\mathcal{O}_{X}, F\right) .
$$

Proof. To motivate, let us assume that $X(\sigma)$ is a Cartier divisor $D$ so that we have an exact sequence

$$
0 \longrightarrow E_{1}^{\prime} \longrightarrow E_{1} \stackrel{\sigma}{\longrightarrow} \mathcal{O}_{X}(-D) \longrightarrow 0
$$

of locally free sheaves. For any coherent sheaf $F$ on $E_{1}(\sigma)$, since $E_{1}^{\prime}$ is a Cartier divisor of $E_{1}(\sigma)$, we have an exact sequence

$$
0 \longrightarrow G \longrightarrow F \longrightarrow \widetilde{F}=\left.F\right|_{E_{1}^{\prime}} \longrightarrow 0
$$

of coherent sheaves on $E_{1}(\sigma)$ with $[G] \in K_{0}\left(\left.E\right|_{X(\sigma)}\right)$ so that $[F]=[\widetilde{F}]+[G]$. We define

$$
0_{E_{1}, \sigma}^{!}[F]=D^{\vee} \cdot 0_{E_{1}^{\prime}}^{!}[\widetilde{F}]+0_{\left.E\right|_{D}}^{!}[G] \in K_{0}(X(\sigma))
$$

where $D^{\vee}: K_{0}(X) \rightarrow K_{0}(D)$ is defined by

$$
D^{\vee} \cdot\left[F^{\prime}\right]=\left[\mathcal{O}_{D}^{\vee} \otimes_{\mathcal{O}_{X}}^{L} F^{\prime}\right]=\sum_{i}(-1)^{i}\left[\operatorname{Tor}_{i}^{X}\left(\mathcal{O}_{D}^{\vee}, F^{\prime}\right)\right]
$$

with $\mathcal{O}_{D}^{\vee}=\left[\mathcal{O}_{X}(-D) \rightarrow \mathcal{O}_{X}\right]^{\vee}=\left[\mathcal{O}_{X} \rightarrow \mathcal{O}_{X}(D)\right]$.

When $X(\sigma)$ is not a Cartier divisor, we let $\rho: \tilde{X} \rightarrow X$ be the blowup of $X$ along $X(\sigma)$ so that we have a surjective homomorphism

$$
\tilde{\sigma}: \widetilde{E}_{1} \longrightarrow \mathcal{O}_{\tilde{X}}(-D)
$$


where $\widetilde{E}_{1}=\rho^{*} E_{1}$ and $D$ is the exceptional divisor. Let $\widetilde{E}_{1}^{\prime}$ be the kernel of $\tilde{\sigma}$. Let $\rho^{\prime}: D \rightarrow X(\sigma)$ denote the restriction of $\rho$ to $D$ and $\tilde{\rho}: \widetilde{E}_{1}^{\prime} \rightarrow E_{1}(\sigma)$ be the map induced by the projection

$$
\widetilde{E}_{1}=E_{1} \times_{X} \tilde{X} \longrightarrow E_{1} .
$$

Let $\tilde{\rho}^{\prime}:\left.\left.\widetilde{E}_{1}^{\prime}\right|_{D} \rightarrow E_{1}\right|_{X(\sigma)}$ denote the restriction of $\tilde{\rho}$ to $\left.\widetilde{E}_{1}\right|_{D}$ and let

$$
\hat{\imath}:\left.E_{1}\right|_{X(\sigma)} \rightarrow E_{1}(\sigma) \text { and } \quad \tilde{\imath}^{\prime}:\left.\widetilde{E}_{1}^{\prime}\right|_{D} \rightarrow \widetilde{E}_{1}^{\prime}
$$

denote the inclusion maps.

For any coherent sheaf $F$ on $E_{1}(\sigma)$, let $\widetilde{F}$ be any coherent sheaf on $\widetilde{E}_{1}^{\prime}$ such that there is an epimorphism

$$
\tilde{\rho}^{*} F \longrightarrow \widetilde{F}
$$

and that

$$
\left.\widetilde{F}\right|_{\left.\widetilde{E}_{1}^{\prime}\right|_{\tilde{X}-D}}=\left.F\right|_{\operatorname{ker}\left(\left.\sigma\right|_{U}\right)}
$$

where we identified $\left.\widetilde{E}_{1}^{\prime}\right|_{\tilde{X}-D}$ with $\operatorname{ker}\left(\left.\sigma\right|_{U}\right)$. For instance, we may choose $\widetilde{F}=\tilde{\rho}^{*} F$.

By adjunction together with (4.7), we have homomorphisms

$$
\eta_{F, \widetilde{F}}: F \longrightarrow \tilde{\rho}_{*} \tilde{\rho}^{*} F \longrightarrow \tilde{\rho}_{*} \widetilde{F} .
$$

Since $F, \tilde{\rho}_{*} \tilde{\rho}^{*} F$ and $\tilde{\rho}_{*} \widetilde{F}$ all coincide over $U$, we have

$$
\left[\operatorname{ker} \eta_{F, \widetilde{F}}\right]-\left[\operatorname{coker} \eta_{F, \widetilde{F}}\right] \in K_{0}\left(\left.E_{1}\right|_{X(\sigma)}\right) \text {. }
$$

Since $\tilde{\rho}$ is an isomorphism over $U$, we have

$$
\left[R^{i} \tilde{\rho}_{*} \widetilde{F}\right] \in K_{0}\left(\left.E_{1}\right|_{X(\sigma)}\right)
$$

for each $i \geq 1$. Let us denote the difference $F-R \tilde{\rho}_{*} \widetilde{F}$ by

$$
R_{F, \widetilde{F}}:=\left[\operatorname{ker} \eta_{F, \widetilde{F}}\right]-\left[\operatorname{coker} \eta_{F, \widetilde{F}}\right]-\sum_{i \geq 1}(-1)^{i}\left[R^{i} \tilde{\rho}_{*} \widetilde{F}\right] \in K_{0}\left(\left.E_{1}\right|_{X(\sigma)}\right) .
$$

We now define the cosection localized Gysin map by

$$
0_{E_{1}, \sigma}^{!}[F]:=\rho_{*}^{\prime}\left(D^{\vee} \cdot 0_{\widetilde{E}_{1}^{\prime}}^{!} \widetilde{F}\right)+0_{\left.E_{1}\right|_{X(\sigma)}}^{R_{F, \widetilde{F}}} \in K_{0}(X(\sigma))
$$

where $D^{\vee} \cdot\left[F^{\prime}\right]=\left[\mathcal{O}_{D}^{\vee} \otimes_{\mathcal{O}_{\tilde{X}}}^{L} F^{\prime}\right]$ for $\mathcal{O}_{D}^{\vee}=\left[\mathcal{O}_{\tilde{X}} \rightarrow \mathcal{O}_{\tilde{X}}(D)\right]$. To complete the proof, we will show the following:

(i) (4.9) is independent of the choice of $\widetilde{F}$.

(ii) If $0 \rightarrow F^{\prime} \rightarrow F \rightarrow F^{\prime \prime} \rightarrow 0$ is an exact sequence of coherent sheaves on $E_{1}(\sigma)$, then

$$
0 ! E_{1, \sigma}[F]=0 ! E_{1, \sigma}\left[F^{\prime}\right]+00_{E_{1}, \sigma}^{!}\left[F^{\prime \prime}\right] .
$$

(iii) (4.3) holds. 
To prove (i), since all $\widetilde{F}$ are quotients of $\tilde{\rho}^{*} F$, it suffices to show

$$
\rho_{*}^{\prime}\left(D^{\vee} \cdot 0_{\widetilde{E}_{1}^{\prime}}^{!} \tilde{\rho}^{*} F\right)+0_{\left.E_{1}\right|_{X(\sigma)}}^{!} R_{F, \tilde{\rho}^{*} F}=\rho_{*}^{\prime}\left(D^{\vee} \cdot 0_{\widetilde{E}_{1}^{\prime}}^{!} \widetilde{F}\right)+0_{\left.E_{1}\right|_{X(\sigma)}}^{!} R_{F, \widetilde{F}}
$$

Let $G$ be the kernel of (4.7) so that we have an exact sequence

$$
0 \longrightarrow \tilde{\imath}_{*}^{\prime} G \longrightarrow \tilde{\rho}^{*} F \longrightarrow \widetilde{F} \longrightarrow 0
$$

with $[G] \in K_{0}\left(\left.\widetilde{E}_{1}^{\prime}\right|_{D}\right)$. Then we have

$$
\begin{aligned}
& \rho_{*}^{\prime}\left(D^{\vee} \cdot 0_{\widetilde{E}_{1}^{\prime}}^{!} \tilde{\rho}^{*} F\right)-\rho_{*}^{\prime}\left(D^{\vee} \cdot 0_{\widetilde{E}_{1}^{\prime}}^{!} \widetilde{F}\right)=\rho_{*}^{\prime}\left(D^{\vee} \cdot 0_{\widetilde{E}_{1}^{\prime}}^{!} \tilde{\imath}_{*}^{\prime} G\right) \\
& =\rho_{*}^{\prime}\left(D^{\vee} \cdot 0_{\left.\widetilde{E}_{1}^{\prime}\right|_{D}}^{!} G\right)=\rho_{*}^{\prime}\left(0_{\left.\widetilde{E}_{1}\right|_{D}}^{!} G\right)=0_{\left.E_{1}\right|_{X(\sigma)}}^{!}\left(\tilde{\rho}_{*}^{\prime}[G]\right) .
\end{aligned}
$$

By 4.12, 4.11) follows once we show

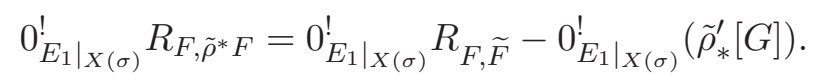

Obviously (4.13) follows from

$$
R_{F, \tilde{\rho}^{*} F}=R_{F, \widetilde{F}}-\tilde{\rho}_{*}^{\prime}[G] \in K_{0}\left(\left.E_{1}\right|_{X(\sigma)}\right) .
$$

To prove (4.14), we consider the long exact sequence

$$
0 \rightarrow \tilde{\rho}_{*}^{\prime} G \rightarrow \tilde{\rho}_{*} \tilde{\rho}^{*} F \rightarrow \tilde{\rho}_{*} \widetilde{F} \rightarrow R^{1} \tilde{\rho}_{*}^{\prime} G \rightarrow R^{1} \tilde{\rho}_{*} \tilde{\rho}^{*} F \rightarrow R^{1} \tilde{\rho}_{*} \widetilde{F} \rightarrow \cdots
$$

and break it into exact sequences as follows:

$$
\begin{gathered}
0 \longrightarrow \tilde{\rho}_{*}^{\prime} G \longrightarrow \tilde{\rho}_{*} \tilde{\rho}^{*} F \longrightarrow H \longrightarrow 0 \\
0 \longrightarrow H \longrightarrow \tilde{\rho}_{*} \widetilde{F} \longrightarrow L \longrightarrow 0 \\
0 \longrightarrow L \longrightarrow R^{1} \tilde{\rho}_{*}^{\prime} G \longrightarrow R^{1} \tilde{\rho}_{*} \tilde{\rho}^{*} F \longrightarrow R^{1} \tilde{\rho}_{*} \widetilde{F} \longrightarrow \cdots
\end{gathered}
$$

Note that (4.18) consists only of sheaves on $\left.E_{1}\right|_{X(\sigma)}$ and we have an equality $(4.19)$

$$
\sum_{i \geq 1}(-1)^{i}\left[R^{i} \tilde{\rho}_{*} \tilde{\rho}^{*} F\right]=\sum_{i \geq 1}(-1)^{i}\left[R^{i} \tilde{\rho}_{*} \widetilde{F}\right]+\sum_{i \geq 1}(-1)^{i}\left[R^{i} \tilde{\rho}_{*}^{\prime} G\right]+[L] \in K_{0}\left(\left.E_{1}\right|_{X(\sigma)}\right) .
$$

From (4.16), we have a commutative diagram of exact sequences

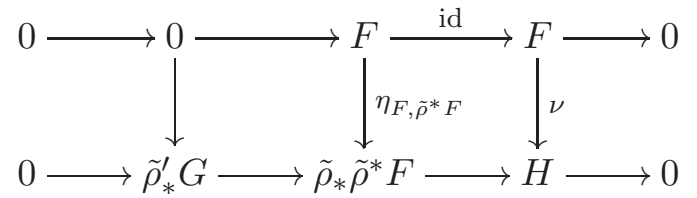

which gives an exact sequence

$$
0 \rightarrow \operatorname{ker}\left(\eta_{F, \tilde{\rho}^{*} F}\right) \rightarrow \operatorname{ker}(\nu) \rightarrow \tilde{\rho}_{*}^{\prime} G \rightarrow \operatorname{coker}\left(\eta_{F, \tilde{\rho}^{*} F}\right) \rightarrow \operatorname{coker}(\nu) \rightarrow 0
$$

of coherent sheaves on $\left.E_{1}\right|_{X(\sigma)}$. Hence we have

$$
\left[\operatorname{ker}\left(\eta_{F, \tilde{\rho}^{*} F}\right)\right]-\left[\operatorname{coker}\left(\eta_{F, \tilde{\rho}^{*} F}\right)\right]=[\operatorname{ker} \nu]-[\operatorname{coker} \nu]-\left[\tilde{\rho}_{*}^{\prime} G\right] \in K_{0}\left(\left.E_{1}\right|_{X(\sigma)}\right) .
$$


From (4.17), we have a commutative diagram of exact sequences

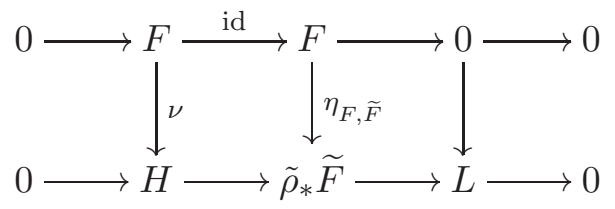

which gives an exact sequence

$$
0 \rightarrow \operatorname{ker} \nu \rightarrow \operatorname{ker}\left(\eta_{F, \widetilde{F}}\right) \rightarrow 0 \rightarrow \operatorname{coker} \nu \rightarrow \operatorname{coker}\left(\eta_{F, \widetilde{F}}\right) \rightarrow L \rightarrow 0
$$

of coherent sheaves on $\left.E_{1}\right|_{X(\sigma)}$. Hence we have

$$
[\operatorname{ker} \nu]-[\operatorname{coker} \nu]=\left[\operatorname{ker}\left(\eta_{F, \widetilde{F}}\right)\right]-\left[\operatorname{coker}\left(\eta_{F, \widetilde{F}}\right)\right]+[L] \in K_{0}\left(\left.E_{1}\right|_{X(\sigma)}\right) \text {. }
$$

Upon adding (4.20) with (4.21) and subtracting (4.19), we obtain

$$
R_{F, \tilde{\rho}^{*} F}=R_{F, \widetilde{F}}-\tilde{\rho}_{*}^{\prime}[G] \in K_{0}\left(\left.E_{1}\right|_{X(\sigma)}\right)
$$

as desired. This proves (i).

Next we prove (ii). Let $0 \rightarrow F^{\prime} \rightarrow F \rightarrow F^{\prime \prime} \rightarrow 0$ be an exact sequence of coherent sheaves on $E_{1}(\sigma)$. Since $\tilde{\rho}^{*}$ is right exact, we have an exact sequence

$$
\tilde{\rho}^{*} F^{\prime} \longrightarrow \tilde{\rho}^{*} F \longrightarrow \tilde{\rho}^{*} F^{\prime \prime} \longrightarrow 0
$$

Let $\widetilde{F}^{\prime}$ be the image of the first arrow and let

$$
\widetilde{F}=\tilde{\rho}^{*} F, \quad \widetilde{F}^{\prime \prime}=\tilde{\rho}^{*} F^{\prime \prime}
$$

so that we have an exact sequence

$$
0 \longrightarrow \widetilde{F}^{\prime} \longrightarrow \widetilde{F} \longrightarrow \widetilde{F}^{\prime \prime} \longrightarrow 0
$$

of coherent sheaves on $\widetilde{E}_{1}^{\prime}$. When applied to the long exact sequence

$$
0 \rightarrow \tilde{\rho}_{*} \widetilde{F}^{\prime} \rightarrow \tilde{\rho}_{*} \widetilde{F} \rightarrow \tilde{\rho}_{*} \widetilde{F}^{\prime \prime} \rightarrow R^{1} \tilde{\rho}_{*} \widetilde{F}^{\prime} \rightarrow R^{1} \tilde{\rho}_{*} \widetilde{F} \rightarrow R^{1} \tilde{\rho}_{*} \widetilde{F}^{\prime \prime} \rightarrow \cdots
$$

associated to (4.23), the same argument that we used above to prove (4.22) gives us the equality

$$
R_{F^{\prime}, \widetilde{F}^{\prime}}+R_{F^{\prime \prime}, \widetilde{F}^{\prime \prime}}=R_{F, \widetilde{F}} \in K_{0}\left(\left.E_{1}\right|_{X(\sigma)}\right) .
$$

On the other hand, (4.23) gives us the equality $[\widetilde{F}]=\left[\widetilde{F}^{\prime}\right]+\left[\widetilde{F}^{\prime \prime}\right] \in K_{0}\left(\widetilde{E}_{1}^{\prime}\right)$ and hence

$$
\rho_{*}^{\prime}\left(D^{\vee} \cdot 0_{\widetilde{E}_{1}^{\prime}}^{!} \widetilde{F}\right)=\rho_{*}^{\prime}\left(D^{\vee} \cdot 0_{\widetilde{E}_{1}^{\prime}}^{!} \widetilde{F}^{\prime}\right)+\rho_{*}^{\prime}\left(D^{\vee} \cdot 0_{\widetilde{E}_{1}^{\prime}} \widetilde{F}^{\prime \prime}\right) \quad \in K_{0}(X(\sigma)) .
$$

Combining (4.24) and (4.25), we obtain (4.10).

Finally we prove (4.3). By its definition (4.8),

$$
\hat{\jmath}_{*} R_{F, \widetilde{F}}=[F]-\tilde{\rho}_{*}[\widetilde{F}] \in K_{0}\left(E_{1}\right)
$$

where $\hat{\jmath}=\jmath \circ \hat{\imath}:\left.E_{1}\right|_{X(\sigma)} \rightarrow E_{1}$ is the inclusion map. Hence

$$
\imath_{*} 0_{\left.E_{1}\right|_{X(\sigma)}} R_{F, \widetilde{F}}=0 !_{E_{1}} \hat{\jmath}_{*} R_{F, \widetilde{F}}=0 !_{E_{1}}[F]-0 !_{E_{1}} \tilde{\rho}_{*}[\widetilde{F}] \in K_{0}(X) .
$$


On the other hand, we have

$$
\imath_{*} \rho_{*}^{\prime}\left(D^{\vee} \cdot 0_{\widetilde{E}_{1}^{\prime}}^{!} \widetilde{F}\right)=\rho_{*} \tilde{\imath}_{*}\left(D^{\vee} \cdot 0_{\widetilde{E}_{1}^{\prime}}^{!} \widetilde{F}\right)=\rho_{*} 0_{\widetilde{E}_{1}}^{!}[\widetilde{F}]=0_{E_{1}}^{!} \tilde{\rho}_{*}[\widetilde{F}]
$$

where $\tilde{\imath}: D \rightarrow \tilde{X}$ is the inclusion. Now (4.3) follows from (4.26), (4.27) and (4.9). This completes the proof.

The following is a basic example.

Example 4.2. Let $X$ be a smooth variety of dimension $n$ and $E$ be a vector bundle on $X$ of rank $n$. Let $\sigma: E \rightarrow \mathcal{O}_{X}$ be a cosection whose zero locus $X(\sigma)$ consists of one simple point $p$. Then

$$
0_{E, \sigma}^{!}\left[\mathcal{O}_{X}\right]=(-1)^{n}\left[\mathcal{O}_{p}\right] \quad \in K_{0}(\{p\}) .
$$

To see it, let $\rho: \tilde{X} \rightarrow X$ be the blowup of $X$ at $p$. Let $D \cong \mathbb{P}^{n-1}$ be the exceptional divisor so that we have an exact sequence

$$
0 \longrightarrow \widetilde{E}^{\prime} \longrightarrow \rho^{*} E \longrightarrow \mathcal{O}_{\tilde{X}}(-D) \longrightarrow 0
$$

whose restriction to the exceptional divisor is

$$
\left.0 \longrightarrow \widetilde{E}^{\prime}\right|_{\mathbb{P}^{n-1}} \longrightarrow \mathcal{O}_{\mathbb{P}^{n-1}}^{\oplus n} \longrightarrow \mathcal{O}_{\mathbb{P}^{n-1}}(1) \longrightarrow 0
$$

so that $\left.\widetilde{E}^{\prime}\right|_{\mathbb{P}^{n-1}}=T_{\mathbb{P} n-1}^{\vee}(1)$. By the Whitney sum formula, $c_{n-1}\left(\left.\widetilde{E}^{\prime}\right|_{\mathbb{P}^{n-1}}\right)=$ $(-1)^{n-1} c_{1}\left(\mathcal{O}_{\mathbb{P}^{n-1}}(1)\right)^{n-1}$ and hence

$$
D^{\vee} \cdot 0_{\widetilde{E}^{\prime}}^{!}\left[\mathcal{O}_{\tilde{X}}\right]=(-1)^{n}\left[\mathcal{O}_{p t}\right]
$$

Since $\rho^{*} \mathcal{O}_{X}=\mathcal{O}_{\tilde{X}}, \rho_{*} \rho^{*} \mathcal{O}_{X}=\rho_{*} \mathcal{O}_{\tilde{X}}=\mathcal{O}_{X}$ and $R^{i} \rho_{*} \mathcal{O}_{\tilde{X}}=0$ for $i \geq 1$, we have $R_{\mathcal{O}_{X}, \mathcal{O}_{\tilde{X}}}=0$ and

$$
0_{E, \sigma}^{!}\left[\mathcal{O}_{X}\right]=\rho_{*}^{\prime}\left(D^{\vee} \cdot 0_{\widetilde{E}^{\prime}}^{!}\left[\mathcal{O}_{\tilde{X}}\right]\right)=(-1)^{n}\left[\mathcal{O}_{p}\right] \in K_{0}(\{p\})
$$

by (4.9).

\section{Cosection localized virtual structure sheaf}

Let $X$ be a Deligne-Mumford stack. Let $\phi: E \rightarrow \mathbb{L}_{X}$ be a perfect obstruction theory and

$$
\sigma: O b_{X}=h^{1}\left(E^{\vee}\right) \longrightarrow \mathcal{O}_{X}
$$

be a homomorphism, called a cosection of the obstruction sheaf $O b_{X}$. Let $X(\sigma)$ be the zero locus of $\sigma$, i.e. the closed substack of $X$ defined by the ideal sheaf $\sigma\left(O b_{X}\right) \subset \mathcal{O}_{X}$.

We assume that $E$ admits a global resolution

$$
\left[E^{-1} \rightarrow E^{0}\right]
$$

by a 2-term complex of locally free sheaves on $X$. The intrinsic normal cone $\mathfrak{C}_{X}$ is canonically embedded into the intrinsic normal sheaf $\mathfrak{N}_{X}$ which in turn embeds into

$$
\mathcal{E}_{X}=h^{1} / h^{0}\left(E^{\vee}\right)=\left[E_{1} / E_{0}\right]
$$


by $h^{1} / h^{0}\left(\phi^{\vee}\right)$ where $E_{i}$ is the dual of $E^{-i}$. The fiber product

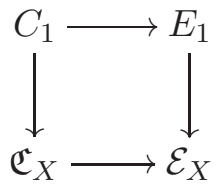

defines a cone $C_{1}$ in $E_{1}$ and we have

$$
\begin{gathered}
{[X]^{\text {vir }}=0_{\mathcal{E}_{X}}^{!}\left[\mathfrak{C}_{X}\right]=0_{E_{1}}^{!}\left[C_{1}\right] \in A_{*}(X),} \\
{\left[\mathcal{O}_{X}^{\text {vir }}\right]=0 !{ }_{E_{1}}\left[\mathcal{O}_{C_{1}}\right]=\left[\mathcal{O}_{X} \otimes_{\mathcal{O}_{E_{1}}}^{L} \mathcal{O}_{C_{1}}\right] \in K_{0}(X) .}
\end{gathered}
$$

In this section, we prove the following.

Theorem 5.1. Suppose a Deligne-Mumford stack $X$ is equipped with a perfect obstruction theory $\phi: E \rightarrow \mathbb{L}_{X}$ and a cosection $\sigma: O b_{X} \rightarrow \mathcal{O}_{X}$ whose zero locus is denoted by $X(\sigma)$. Assume $E$ admits a global resolution $\left[E^{-1} \rightarrow E^{0}\right]$ by locally free sheaves whose dual is denoted by $\left[E_{0} \rightarrow E_{1}\right]$ and let $C_{1}=\mathfrak{C}_{X} \times_{\left[E_{1} / E_{0}\right]} E_{1}$ be the lift of the intrinsic normal cone $\mathfrak{C}_{X}$ to $E_{1}$, whose support is contained in $E_{1}(\sigma)$ by [19, §4]. We define the cosection localized virtual structure sheaf to be

$$
\left[\mathcal{O}_{X, \text { loc }}^{\mathrm{vir}}\right]=0_{E_{1}, \underline{\sigma}}^{!}\left[\mathcal{O}_{C_{1}}\right] \in K_{0}(X(\sigma))
$$

where $\underline{\sigma}: E_{1} \rightarrow h^{1}\left(E^{\vee}\right)=O b_{X} \rightarrow \mathcal{O}_{X}$ is the composition of the quotient map $E_{1} \rightarrow h^{1}\left(E^{\vee}\right)$ with $\sigma$. It satisfies

$$
\imath_{*}\left[\mathcal{O}_{X, \mathrm{loc}}^{\mathrm{vir}}\right]=\left[\mathcal{O}_{X}^{\mathrm{vir}}\right] \in K_{0}(X)
$$

where $\imath: X(\sigma) \rightarrow X$ denotes the inclusion.

We will further show that the cosection localized virtual structure sheaf is deformation invariant and independent of all the choices such as a resolution $\left[E^{-1} \rightarrow E^{0}\right]$ of the perfect obstruction theory on $X$.

Proof. Since $\left[\mathcal{O}_{X}^{\text {vir }}\right]=0 !{ }_{E_{1}}\left[\mathcal{O}_{C_{1}}\right]$, by (4.3) , we have

$$
\imath_{*}\left[\mathcal{O}_{X, \mathrm{loc}}^{\mathrm{vir}}\right]=\imath_{*} 0_{E_{1}, \underline{\sigma}}^{{ }_{(}}\left[\mathcal{O}_{C_{1}}\right]=0_{E_{1}}^{!}\left[\mathcal{O}_{C_{1}}\right]=\left[\mathcal{O}_{X}^{\mathrm{vir}}\right] \in K_{0}(X)
$$

as desired.

Proposition 5.2. The cosection localized virtual structure sheaf $\left[\mathcal{O}_{X, \text { loc }}^{\mathrm{vir}}\right] \in$ $K_{0}(X(\sigma))$ is independent of the choice of a global resolution $\left[E^{-1} \rightarrow E^{0}\right]$ of the perfect obstruction theory $E$ of $X$.

Proof. By the proof of [2, Proposition 5.3], it suffices to consider the case where the two resolutions are related by surjective homomorphisms

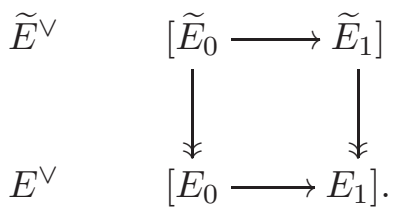


If we let $C_{1} \subset E_{1}$ be the pullback of the intrinsic normal cone $\mathfrak{C}_{X} \subset E_{1} / E_{0}$ of $X$ to $E_{1}$, then $\widetilde{C}_{1}=C_{1} \times_{E_{1}} \widetilde{E}_{1}$ is the pullback of $\mathfrak{C}_{X}$ to $\widetilde{E}_{1}$. Then $\left[\mathcal{O}_{\widetilde{C}_{1}}\right]=q^{*}\left[\mathcal{O}_{C_{1}}\right]$ where $q: \widetilde{E}_{1} \rightarrow E_{1}$ denotes the projection. By the definition of $0_{E_{1}, \sigma}^{!}$above, it is straightforward to see that

$$
0_{E_{1}, \underline{\sigma}}^{!}\left[\mathcal{O}_{C_{1}}\right]=0_{\widetilde{E}_{1}, \underline{\tilde{\sigma}}}^{!}\left[\mathcal{O}_{\widetilde{C}_{1}}\right]
$$

where $\underline{\tilde{\sigma}}$ is the composition $\widetilde{E}_{1} \rightarrow E_{1} \stackrel{\underline{\sigma}}{\rightarrow} \mathcal{O}_{X}$.

Remark 5.3. Exactly the same holds in the algebraic cobordism group of $X$ instead of the algebraic K-group $K_{0}(X)$. See [35] for a virtual fundamental class in the algebraic cobordism group of $X$. We have the cosection localized virtual class $[X]_{\Omega, \text { loc }}^{\mathrm{vir}}$ in the algebraic cobordism group of $X(\sigma)$. Details will appear in a subsequent paper.

Definition 5.4. Let $X$ be a Deligne-Mumford stack equipped with a perfect obstruction theory $\phi: E \rightarrow \mathbb{L}_{X}$ and a cosection $\sigma: O b_{X}=h^{1}\left(E^{\vee}\right) \rightarrow \mathcal{O}_{X}$. Let $\left[\mathcal{O}_{X, \text { loc }}^{\mathrm{vir}}\right] \in K_{0}(X(\sigma))$ be the cosection localized virtual structure sheaf of $X$. Suppose the zero locus $X(\sigma)$ of the cosection $\sigma$ is proper. The cosection localized virtual Euler characteristic of a class $\beta \in K^{0}(X)$ is defined as

$$
\chi_{\mathrm{loc}}^{\mathrm{vir}}(X, \beta)=\chi\left(X(\sigma), \beta \cdot \mathcal{O}_{X, \mathrm{loc}}^{\mathrm{vir}}\right)=\sum_{i}(-1)^{i} \operatorname{dim} H^{i}\left(X(\sigma), \beta \cdot \mathcal{O}_{X, \mathrm{loc}}^{\mathrm{vir}}\right) .
$$

We next prove that the cosection localized virtual structure sheaf is deformation invariant. Let $X \rightarrow S$ be a morphism of stacks, where $X$ is a Deligne-Mumford stack and $S$ is a smooth Artin stack. Let $v: Z \rightarrow W$ be a regular embedding of schemes that fits into a Cartesian square

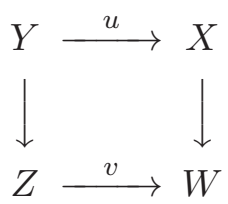

Suppose we have relative perfect obstruction theories $\phi: E \rightarrow \mathbb{L}_{X / S}$ and $\phi^{\prime}: E^{\prime} \rightarrow \mathbb{L}_{Y / S}$ that fit into a morphism of distinguished triangles

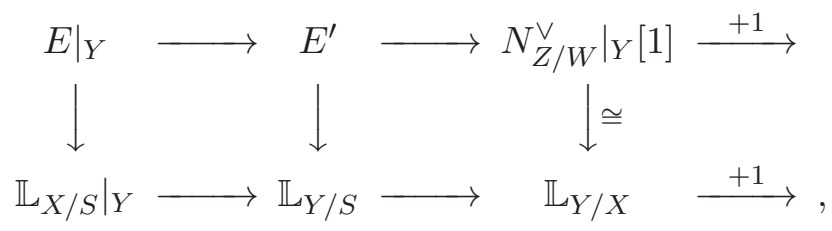

where $N_{Z / W}$ is the normal bundle of $Z$ in $W$.

As above, we assume the (relative) perfect obstruction theory $E$ admits a global resolution

$$
\left[E^{-1} \rightarrow E^{0}\right]
$$


by locally free sheaves $E^{-1}$ and $E^{0}$ on $X$. Since $E^{\prime}$ is the cone of the morphism $\left.\left.N_{Z / W}^{\vee}\right|_{Y} \rightarrow E\right|_{Y}, E^{\prime}$ has the global resolution

$$
\left[\left.\left.\left.E^{-1}\right|_{Y} \oplus N_{Z / W}^{\vee}\right|_{Y} \rightarrow E^{0}\right|_{Y}\right] .
$$

From the distinguished triangle $\left.\mathbb{L}_{S}\right|_{X} \rightarrow \mathbb{L}_{X} \rightarrow \mathbb{L}_{X / S} \rightarrow \cdots$, we find that

$$
\left[\left.E^{-1} \rightarrow E^{0} \oplus T_{S}^{\vee}\right|_{X}\right]
$$

is an (absolute) perfect obstruction theory of $X$. Similarly,

$$
\left[\left.\left.\left.\left.E^{-1}\right|_{Y} \oplus N_{Z / W}^{\vee}\right|_{Y} \rightarrow E^{0}\right|_{Y} \oplus T_{S}^{\vee}\right|_{Y}\right]
$$

is an (absolute) perfect obstruction theory of $Y$. Recall that the (absolute) obstruction sheaf $O b_{X}$ of $X$ is the cokernel of the dual of (5.7) and the obstruction sheaf $O b_{Y}$ of $Y$ is that of the dual of (5.8). Hence we have an exact sequence

$$
\left.\left.N_{Z / W}\right|_{Y} \longrightarrow O b_{Y} \longrightarrow O b_{X}\right|_{Y} \longrightarrow 0 .
$$

Let $\sigma: O b_{X} \rightarrow \mathcal{O}_{X}$ be a cosection for $X$ and let

$$
\sigma^{\prime}:\left.O b_{Y} \rightarrow O b_{X}\right|_{Y} \stackrel{\left.\sigma\right|_{Y}}{\longrightarrow} \mathcal{O}_{Y}
$$

be the induced cosection for $Y$. Let $\mathcal{O}_{X, \text { loc }}^{\text {vir }}\left(\right.$ resp. $\left.\mathcal{O}_{Y, \text { loc }}^{\text {vir }}\right)$ be the cosection localized virtual structure sheaf of $X$ (resp. $Y$ ) with respect to the perfect obstruction theory (5.7) of $X$ (resp. (5.8) of $Y$ ). Let $X(\sigma)$ (resp. $Y(\sigma)$ ) denote the zero locus of the cosection $\sigma$ and (resp. $\sigma^{\prime}$ ). Then by construction, $Y(\sigma)=X(\sigma) \times_{X} Y$ which fits into the Cartesian square

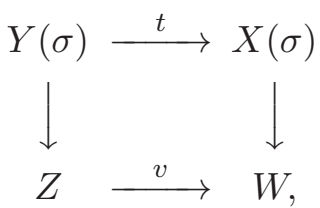

since $Y=Z \times_{W} X$. We then have the Gysin map

$$
v^{!}: K_{0}(X(\sigma)) \rightarrow K_{0}(Y(\sigma))
$$

defined by

$$
v^{!}\left[\mathcal{O}_{A}\right]=0_{N_{Z / W}}^{!}\left[\mathcal{O}_{C_{Y(\sigma) \cap A / A}}\right] \in K_{0}(Y(\sigma)), \quad A \subset X(\sigma) .
$$

In fact, if we choose a finite locally free resolution $\mathcal{O}_{Z}^{W}$ of $v_{*} \mathcal{O}_{Z}$ on $W$, then the Gysin map $v^{!}$is given by the tensor product

$$
v^{!}[F]=\left[\left.\mathcal{O}_{Z}^{W}\right|_{X(\sigma)} \otimes_{\mathcal{O}_{X(\sigma)}} F\right], \quad F \in K_{0}(X(\sigma))
$$

by [29, Remark 1].

To avoid discussion about K-theory of Artin stacks, let us assume that there is a smooth morphism $M \rightarrow S$ and a closed embedding $X \subset M$ over $S$. This is always possible if, for instance, $X \rightarrow S$ is quasi-projective.

Proposition 5.5. Under the above assumptions, we have

$$
v^{!}\left[\mathcal{O}_{X, \text { loc }}^{\mathrm{vir}}\right]=\left[\mathcal{O}_{Y, \text { loc }}^{\mathrm{vir}}\right] \in K_{0}(Y(\sigma)) \text {. }
$$


Proof. The proof essentially follows from that of [19, Theorem 5.2], using the work of [24]. We outline the arguments. By [13, there is a deformation $\mathcal{M} \rightarrow \mathbb{P}^{1}$ whose fibers are $M$ except the central fiber $C_{X / M}$ which is the normal cone of $X$ in $M$. In [24, below (7)], the authors constructed a double deformation space over $\mathbb{P}^{1} \times \mathbb{P}^{1}$, that is the deformation of $\mathcal{M}$ to the normal cone $C_{Y \times \mathbb{P}^{1} / \mathcal{M}}$ of $Y \times \mathbb{P}^{1}$. We denote this double deformation space by $\mathcal{W}$.

By its construction, $\mathcal{W}$ is flat over $\mathbb{P}^{1} \times \mathbb{P}^{1}-\{(0,0)\}$, where $(0,0) \in \mathbb{P}^{1} \times \mathbb{P}^{1}$ is the special point having the following properties: The fiber of $\mathcal{W}$ over $(1,0) \in \mathbb{P}^{1} \times \mathbb{P}^{1}$ is $C_{Y / M}$; the flat specialization along $\mathbb{P}^{1} \times\{0\}($ over $(0,0)$ ) is $C_{Y / C_{X / M}}$.

Because $X$ is of finite type, an easy argument shows that we can find a smooth birational model $U \rightarrow \mathbb{P}^{1} \times \mathbb{P}^{1}$, isomorphic over $\mathbb{P}^{1} \times \mathbb{P}^{1}-\{(0,0)\}$, so that

$$
\mathcal{W} \times \mathbb{P}^{1} \times \mathbb{P}^{1}\left(\mathbb{P}^{1} \times \mathbb{P}^{1}-\{(0,0)\}\right) \times_{\mathbb{P}^{1} \times \mathbb{P}^{1}} U
$$

extends to a family $\tilde{\mathcal{W}} \subset \mathcal{W} \times_{\mathbb{P}^{1} \times \mathbb{P}^{1}} U$, flat over $U$. Consequently, we can find a chain $\Sigma=\cup_{i=1}^{n} \Sigma_{i}$ of $\mathbb{P}^{1}$ 's in $U$, lying over $\mathbb{P}^{1} \times\{0\}$, and two points $a \in \Sigma_{1}$ and $b \in \Sigma_{n}$ so that

$$
\left.\tilde{\mathcal{W}}\right|_{a}=C_{Y / M},\left.\quad \tilde{\mathcal{W}}\right|_{b}=C_{Y / C_{X / M}}
$$

We thus obtain a rational equivalence

$$
\left[C_{Y / M}\right]=\left[C_{Y / C_{X / M}}\right] .
$$

Recall that (5.7) and (5.8) are perfect obstruction theories for $X$ and $Y$ respectively. Let $E_{i}$ denote the dual of $E^{-i}$ for $i=0,1$. Since the intrinsic normal cone is $\mathfrak{C}_{X}=C_{X / M} /\left.T_{M}\right|_{X}$, the cone $C_{X}:=C_{1}=\mathfrak{C}_{X} \times \mathcal{E}_{X} E_{1}$ equals

$$
C_{X / M} \times_{X}\left(\left.E_{0} \oplus T_{S}\right|_{X}\right) /\left.T_{M}\right|_{X} \subset E_{1}(\underline{\sigma})
$$

where $\underline{\sigma}: E_{1} \rightarrow O b_{X} \stackrel{\sigma}{\longrightarrow} \mathcal{O}_{X}$ is the cosection of $E_{1}$ induced by $\sigma$. Hence we have

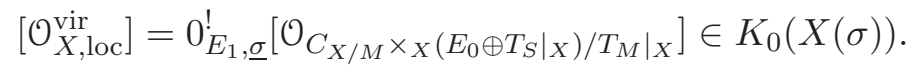

By (5.9), the restriction $\underline{\sigma}^{\prime}:\left.E_{1}\right|_{Y} \rightarrow \mathcal{O}_{Y}$ of $\underline{\sigma}$ factors through $\sigma^{\prime}: O b_{Y} \rightarrow$ $\mathcal{O}_{Y}$ defined above. By definition, we have the equality $\left.E_{1}(\underline{\sigma})\right|_{Y}=\left.E_{1}\right|_{Y}\left(\underline{\sigma}^{\prime}\right)$ and a Cartesian diagram

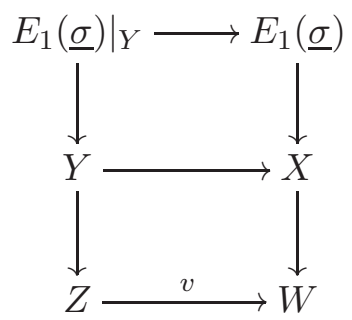


Similarly to the case of $X$ above, the cone $C_{Y}=\mathfrak{C}_{Y} \times \mathcal{E}_{Y}\left(\left.\left.E_{1}\right|_{Y} \oplus N_{Z / W}\right|_{Y}\right)$ equals

$$
C_{Y / M} \times_{Y}\left(\left.\left.E_{0}\right|_{Y} \oplus T_{S}\right|_{Y}\right) /\left.\left.\left.T_{M}\right|_{Y} \subset E_{1}(\underline{\sigma})\right|_{Y} \oplus N_{Z / W}\right|_{Y}
$$

so that

$$
\begin{aligned}
& {\left[\mathcal{O}_{Y, \text { loc }}^{\mathrm{vir}}\right]=0_{\left.\left.E_{1}\right|_{Y} \oplus N_{Z / W}\right|_{Y, \underline{\sigma}^{\prime}}}\left[\mathcal{O}_{\left.C_{Y / M} \times_{Y}\left(\left.\left.E_{0}\right|_{Y} \oplus T_{S}\right|_{Y}\right) /\left.T_{M}\right|_{Y}\right]}\right]} \\
& =0_{\left.E_{1}\right|_{Y}, \underline{\sigma}^{\prime}} 00_{\left.N_{Z / W}\right|_{Y}}\left[\mathcal{O}_{C_{Y / M} \times_{Y}\left(\left.\left.E_{0}\right|_{Y} \oplus T_{S}\right|_{Y}\right) /\left.T_{M}\right|_{Y}}\right]
\end{aligned}
$$

since $\left.N_{Z / W}\right|_{Y}$ lies in the kernel of $\sigma^{\prime}$ by (5.9).

It was proved in $[19, \S 5]$ that the rational equivalence (5.12) takes place in the kernel of a cosection extended over the double deformation space. Therefore (5.14) equals

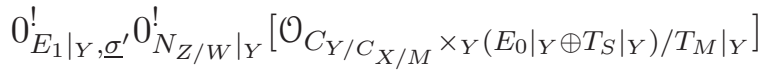

$$
\begin{aligned}
& =0_{\left.E_{1}\right|_{Y}, \underline{\sigma}^{\prime}} v^{!}\left[\mathcal{O}_{C_{X / M} \times X}\left(\left.E_{0} \oplus T_{S}\right|_{X}\right) /\left.T_{M}\right|_{X}\right] .
\end{aligned}
$$

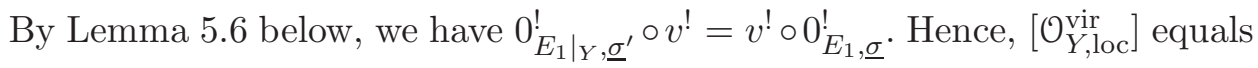

$$
v^{!} 0_{E_{1}, \underline{\sigma}}^{!}\left[\mathcal{O}_{C_{X / M} \times{ }_{X}\left(\left.E_{0} \oplus T_{S}\right|_{X}\right) /\left.T_{M}\right|_{X}}\right]=v^{!}\left[\mathcal{O}_{X, \text { loc }}^{\text {vir }}\right]
$$

by (5.13). This proves the proposition.

It remains to prove the following.

Lemma 5.6. With the notation as above, $0_{\left.E_{1}\right|_{Y, \underline{\sigma}^{\prime}}} \circ v^{!}=v^{!} \circ 0_{E_{1}, \underline{\sigma}}$.

Proof. Let $\rho: \tilde{X} \rightarrow X$ denote the blowup of $X$ along $X(\sigma)$ with exceptional divisor $D$ so that we have an exact sequence

$$
0 \longrightarrow \widetilde{E}_{1}^{\prime} \longrightarrow \widetilde{E}_{1} \longrightarrow \mathcal{O}_{\tilde{X}}(-D) \longrightarrow 0
$$

where $\widetilde{E}_{1}=\rho^{*} E_{1}$. Let $A \subset E_{1}(\sigma)$ be an integral substack. If $\left.A \subset E_{1}\right|_{X(\sigma)}$, the lemma simply says $v^{!} \circ 0_{E_{1}}^{!}=0_{\left.E_{1}\right|_{Y}}^{!} \circ v^{!}$which is straightforward since both $v^{!}$and $0 ! E_{1}$ are tensor products (cf. (5.11) $)$. If $\left.A \not \subset E_{1}\right|_{X(\sigma)}$, let $\tilde{A} \subset \widetilde{E}_{1}^{\prime}$ be the proper transform of $A$ so that

$$
0_{E_{1}, \underline{\sigma}}^{!}\left[\mathcal{O}_{A}\right]=\rho_{*}^{\prime}\left(D^{\vee} \cdot 0_{\widetilde{E}_{1}^{\prime}}^{!}\left[\mathcal{O}_{\tilde{A}}\right]\right)+0_{\left.E_{1}\right|_{X(\sigma)}}^{!}\left[\mathcal{O}_{A} \rightarrow \rho_{*} \mathcal{O}_{\tilde{A}}\right] .
$$

Since $v^{!}\left[\mathcal{O}_{A}\right]=\left[\left.\mathcal{O}_{Z}^{W}\right|_{E_{1}(\underline{\sigma})} \otimes \mathcal{O}_{A}\right]$ where $\mathcal{O}_{Z}^{W}$ is a finite locally free resolution of $v_{*} \mathcal{O}_{Z}$ on $W$, we have

$0_{\left.E_{1}\right|_{Y}, \underline{\sigma}^{\prime}}^{!} v^{!}\left[\mathcal{O}_{A}\right]=\rho_{*}^{\prime}\left(D^{\vee} \cdot 0_{\widetilde{E}_{1}^{\prime}}^{!}\left[\left.\mathcal{O}_{Z}^{W}\right|_{\widetilde{E}_{1}^{\prime}} \otimes \mathcal{O}_{\tilde{A}}\right]\right)+0_{\left.E_{1}\right|_{X(\sigma)}}^{!}\left[\left.\mathcal{O}_{Z}^{W}\right|_{\left.E_{1}\right|_{X(\sigma)}} \otimes\left[\mathcal{O}_{A} \rightarrow \rho_{*} \mathcal{O}_{\tilde{A}}\right]\right]$.

Since $\mathcal{O}_{Z}^{W}$ is a complex of locally free sheaves on $W$ and the Gysin maps are tensor products, we can pull out $\mathcal{O}_{Z}^{W}$ to obtain

$$
\begin{aligned}
& 0_{\left.E_{1}\right|_{Y}, \underline{\sigma}^{\prime}} v^{!}\left[\mathcal{O}_{A}\right]=\left.\mathcal{O}_{Z}^{W}\right|_{X(\sigma)} \otimes \rho_{*}^{\prime}\left(D^{\vee} \cdot 0_{\widetilde{E}_{1}^{\prime}}^{!}\left[\mathcal{O}_{\tilde{A}}\right]\right)+\left.\mathcal{O}_{Z}^{W}\right|_{X(\sigma)} \otimes 0_{\left.E_{1}\right|_{X(\sigma)}}^{!}\left[\mathcal{O}_{A} \rightarrow \rho_{*} \mathcal{O}_{\tilde{A}}\right] \\
& =\left.\mathcal{O}_{Z}^{W}\right|_{X(\sigma)} \otimes 0_{E_{1}, \underline{\sigma}}^{!}\left[\mathcal{O}_{A}\right]=v 0^{!}{ }_{E_{1}, \underline{\sigma}}\left[\mathcal{O}_{A}\right]
\end{aligned}
$$

as desired. 
As an application of Proposition 5.5. we will prove a principle of conservation of numbers for the cosection localized virtual Euler characteristics. Let $t \in \mathbb{P}^{1}$ be a closed point and $\pi: X \rightarrow \mathbb{P}^{1}$ be a morphism of Deligne-Mumford stacks, equipped with a relative perfect obstruction theory

$$
\phi: E \rightarrow \mathbb{L}_{X / \mathbb{P}^{1}} .
$$

Let $\sigma: O b_{X / \mathbb{P}^{1}}=h^{1}\left(E^{\vee}\right) \rightarrow \mathcal{O}_{X}$ be a cosection of the relative obstruction sheaf whose zero locus is denoted by $X(\sigma)$. We assume that $X(\sigma)$ is proper over $\mathbb{P}^{1}$ although $X$ may not be proper over $\mathbb{P}^{1}$. The restriction

$$
\phi_{t}: E_{t} \longrightarrow \mathbb{L}_{X_{t}}
$$

of $\phi$ to $X_{t}=t \times_{\mathbb{P}^{1}} X$ with $E_{t}=\left.E\right|_{X_{t}}$ is a perfect obstruction theory of $X_{t}$ and we have an absolute perfect obstruction theory

$$
\bar{\phi}: \bar{E} \longrightarrow \mathbb{L}_{X}
$$

of $X$ defined by the commutative diagram

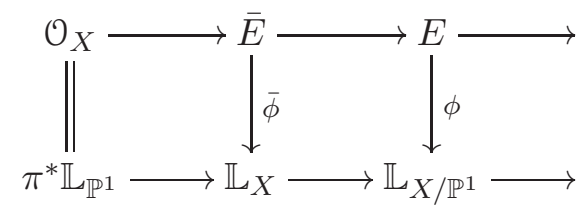

of distinguished triangles. The first row of (5.15) gives us an exact sequence

$$
\mathcal{O}_{X} \longrightarrow O b_{X / \mathbb{P}^{1}}=h^{1}\left(E^{\vee}\right) \longrightarrow O b_{X}=h^{1}\left(\bar{E}^{\vee}\right) \longrightarrow 0 .
$$

We further assume that the cosection $\sigma: O b_{X / \mathbb{P}^{1}} \rightarrow \mathcal{O}_{X}$ descends to a cosection

$$
\bar{\sigma}: O b_{X} \rightarrow \mathcal{O}_{X}
$$

so that we have the cosection localized virtual structure sheaf $\left[\mathcal{O}_{X, \text { loc }}^{\text {vir }}\right] \in$ $K_{0}(X(\bar{\sigma}))$. Likewise, the homomorphism $\sigma_{t}: O b_{X_{t}} \rightarrow \mathcal{O}_{X_{t}}$ induced by $\sigma$ gives us the cosection localized virtual structure sheaf $\left[\mathcal{O}_{X_{t}, \text { loc }}^{\text {vir }}\right] \in K_{0}\left(X_{t}\left(\sigma_{t}\right)\right)$.

Corollary 5.7. Under the above assumptions, for any $\beta \in K^{0}(X(\bar{\sigma}))$

$$
\chi_{\mathrm{loc}}^{\mathrm{vir}}\left(X_{t}, \imath_{t}^{*} \beta\right)=\chi\left(X_{t}\left(\sigma_{t}\right), \mathcal{O}_{X_{t}, \text { loc }}^{\mathrm{vir}} \cdot \imath_{t}^{*} \beta\right)=\chi\left(X(\bar{\sigma}), \imath_{t *} \mathcal{O}_{X_{t}, \text { loc }}^{\mathrm{vir}} \cdot \beta\right)
$$

is independent of $t \in \mathbb{P}^{1}$, where $\imath_{t}: X_{t}\left(\sigma_{t}\right) \hookrightarrow X(\bar{\sigma})$.

Proof. By Proposition 5.5, we have

$$
t^{!}\left[\mathcal{O}_{X, \mathrm{loc}}^{\mathrm{vir}}\right]=\left[\mathcal{O}_{X_{t}, \mathrm{loc}}^{\mathrm{vir}}\right] \in K_{0}\left(X_{t}\left(\sigma_{t}\right)\right)
$$

where $t:\{t\} \rightarrow \mathbb{P}^{1}$ is the inclusion. Then we have

$$
\imath_{t *}\left[\mathcal{O}_{X_{t}, \text { loc }}^{\mathrm{vir}}\right]=\imath_{t *} t^{!}\left[\mathcal{O}_{X, \mathrm{loc}}^{\mathrm{vir}}\right] \in K_{0}(X(\bar{\sigma})) .
$$

Since $\left[\mathcal{O}_{t}\right] \in K_{0}\left(\mathbb{P}^{1}\right)$ is independent of $t \in \mathbb{P}^{1}, \imath_{t *} t^{!}\left[\mathcal{O}_{X, \text { loc }}^{\text {vir }}\right]$ is independent of $t$. Hence

$$
\chi\left(X(\bar{\sigma}), \imath_{t *} \mathcal{O}_{X_{t}, \text { loc }}^{\mathrm{vir}} \cdot \beta\right), \quad \beta \in K^{0}(X(\bar{\sigma}))
$$

is independent of $t$ as desired. 
Another way to prove Corollary 5.7 is to use a cosection localized RiemannRoch as outlined below. For schemes, we have the following cosection localized version of virtual Grothendieck-Riemann-Roch (cf. [12, Theorem $3.3])$.

Theorem 5.8. Let $f: X \rightarrow Y$ be a morphism of schemes with $Y$ smooth. Let $\phi: E \rightarrow \mathbb{L}_{X}$ be a perfect obstruction theory of $X$ and $\sigma: O b_{X} \rightarrow \mathcal{O}_{X}$ be a cosection. Suppose the restriction $f^{\prime}: X(\sigma) \rightarrow Y$ of $f$ to the zero locus $X(\sigma)$ of the cosection $\sigma$ is proper. Let $T_{X}^{\mathrm{vir}}=\left[E_{0}\right]-\left[E_{1}\right] \in K^{0}(X)$ be the virtual tangent bundle where $\left[E_{0} \rightarrow E_{1}\right]$ is the dual of $E$. Then for $\beta \in K^{0}(X)$, we have

$$
\operatorname{ch}\left(f_{*}^{\prime}\left(\beta \cdot \mathcal{O}_{X, \mathrm{loc}}^{\mathrm{vir}}\right)\right) \operatorname{td}\left(T_{Y}\right) \cap[Y]=f_{*}^{\prime}\left(\operatorname{ch}(\beta) \operatorname{td}\left(T_{X}^{\mathrm{vir}}\right) \cap[X]_{\mathrm{loc}}^{\mathrm{vir}}\right) .
$$

In particular, if $Y$ is a point and $X(\sigma)$ is proper, the cosection localized virtual Euler characteristic of $\beta$ is

$$
\chi_{\mathrm{loc}}^{\mathrm{vir}}(X, \beta)=\int_{[X]_{\mathrm{loc}}^{\mathrm{vir}}} \operatorname{ch}(\beta) \operatorname{td}\left(T_{X}^{\mathrm{vir}}\right) .
$$

For a quasi-projective Deligne-Mumford stack $X$, we choose a smooth projective Deligne-Mumford stack $M$ and a closed immersion $X \hookrightarrow M$. Then (5.17) is

$$
\chi_{\mathrm{loc}}^{\mathrm{vir}}\left(X_{t}, \imath_{t}^{*} \beta\right)=\chi\left(M, \jmath_{*} \imath_{t *} \mathcal{O}_{X_{t}, \mathrm{loc}}^{\mathrm{vir}} \cdot \beta\right), \quad \beta \in K^{0}(M)
$$

where $\jmath: X(\bar{\sigma}) \hookrightarrow X \hookrightarrow M$ is the inclusion. Applying the KawasakiRiemann-Roch for the smooth Deligne-Mumford stack $M$ together with (5.18), we can express the right side of (5.20) in terms of cosection localized virtual integrals on inertia substacks $X_{\mu}$. Since the cosection localized virtual fundamental classes are deformation invariant by [19], we find that the left side of (5.20) is independent of $t$.

We complete this section with a proof of (5.18).

Proof of Theorem 5.8. The theorem is proved by copying the proof of $[12$, Theorem 3.3] line by line, if we replace the ordinary Gysin map $0_{E_{1}}^{!}$by the cosection localized Gysin map $0_{E_{1}, \sigma}^{!}$. Perhaps the only nontrivial fact to be checked is the identity

$$
\tau_{X(\sigma)}\left(0_{E_{1}, \sigma}^{!}[F]\right)=\operatorname{td}\left(-E_{1}\right) \cap 0 ! E_{E_{1}, \sigma}\left(\tau_{E_{1}(\sigma)}[F]\right)
$$

for a coherent sheaf $F$ on $E_{1}(\sigma)$.

Recall that the cosection localized Gysin map for Chow groups

$$
0 ! E_{1}, \sigma: A_{*}\left(E_{1}(\sigma)\right) \rightarrow A_{*}(X(\sigma))
$$

is defined in 19 by

$$
0_{E_{1}, \sigma}^{!}(\xi)=\rho_{*}^{\prime}\left(-D \cdot 0_{\widetilde{E}_{1}^{\prime}}^{!}(\zeta)\right)+0_{\left.E_{1}\right|_{X(\sigma)}}^{!}(\eta)
$$

whenever $\xi=\tilde{\rho}_{*} \zeta+\hat{\imath}_{*} \eta$ for $\zeta \in A_{*}\left(\widetilde{E}_{1}^{\prime}\right)$ and $\eta \in A_{*}\left(\left.E_{1}\right|_{X(\sigma)}\right)$. Here $\hat{\imath}$ : $\left.E\right|_{X(\sigma)} \rightarrow E_{1}(\sigma)$ is the inclusion and $\rho: \tilde{X} \rightarrow X$ is the blowup of $X$ along 
$X(\sigma)$ while $\rho^{\prime}: D \rightarrow X(\sigma)$ is the restriction of $\rho$ to the exceptional divisor $D$ as in $\$$.

For any choice of $\widetilde{F}$ as in (4.7), by (4.8), we have $F=R \tilde{\rho}_{*} \widetilde{F}+R \hat{\imath}_{*} R_{F, \widetilde{F}}$ with $[\widetilde{F}] \in K_{0}\left(\widetilde{E}_{1}^{\prime}\right)$ and $\left[R_{F, \widetilde{F}}\right] \in K_{0}\left(\left.E_{1}\right|_{X(\sigma)}\right)$. Hence, we have

$$
\begin{gathered}
\tau_{E_{1}(\sigma)}[F]=\tau_{E_{1}(\sigma)} R \tilde{\rho}_{*} \widetilde{F}+\tau_{E_{1}(\sigma)} R \hat{\imath}_{*} R_{F, \widetilde{F}} \\
=\tilde{\rho}_{*} \tau_{\widetilde{E}_{1}^{\prime}}[\widetilde{F}]+\hat{\imath}_{*} \tau_{\left.E_{1}\right|_{X(\sigma)}} R_{F, \widetilde{F}} .
\end{gathered}
$$

By (5.22), we thus have

$$
0_{E_{1}, \sigma}^{!}\left(\tau_{E_{1}(\sigma)}[F]\right)=\rho_{*}^{\prime}\left(-D \cdot 0_{\widetilde{E}_{1}^{\prime}}^{!}\left(\tau_{\widetilde{E}_{1}^{\prime}}[\widetilde{F}]\right)\right)+0_{\left.E_{1}\right|_{X(\sigma)}}^{{ }^{\prime}}\left(\tau_{\left.E_{1}\right|_{X(\sigma)}} R_{F, \widetilde{F}}\right) .
$$

By (4.9) and (5.24), we have

$$
\begin{aligned}
& \tau_{X(\sigma)} 0_{E_{1}, \sigma}^{!}[F]=\tau_{X(\sigma)} \rho_{*}^{\prime}\left(D^{\vee} \cdot 0_{\widetilde{E}_{1}^{\prime}}^{!} \widetilde{F}\right)+\tau_{X(\sigma)} 0_{\left.E_{1}\right|_{X(\sigma)}}^{R_{F, \widetilde{F}}} \\
& =\rho_{*}^{\prime} \tau_{D}\left(\mathcal{O}_{D}^{\vee} \otimes 0_{\widetilde{E}_{1}^{\prime}}^{!} \widetilde{F}\right)+\operatorname{td}\left(-E_{1}\right) 0_{\left.E_{1}\right|_{X(\sigma)}}^{!}\left(\tau_{\left.E_{1}\right|_{X(\sigma)}} R_{F, \widetilde{F}}\right) \\
& =\rho_{*}^{\prime} \operatorname{ch}\left(\mathcal{O}_{D}^{\vee}\right) \tau_{D}\left(0_{\widetilde{E}_{1}^{\prime}}^{!} \widetilde{F}\right)+\operatorname{td}\left(-E_{1}\right) 0_{\left.E_{1}\right|_{X(\sigma)}}^{{ }^{\prime}}\left(\tau_{\left.E_{1}\right|_{X(\sigma)}} R_{F, \widetilde{F}}\right) \\
& =\rho_{*}^{\prime} \operatorname{ch}\left(\mathcal{O}_{D}^{\vee}\right) \operatorname{td}\left(-\widetilde{E}_{1}^{\prime}\right) 0_{\widetilde{E}_{1}^{\prime}}^{!}\left(\tau_{\widetilde{E}_{1}^{\prime}} \widetilde{F}\right)+\operatorname{td}\left(-E_{1}\right) 0_{\left.E_{1}\right|_{X(\sigma)}}^{!}\left(\tau_{\left.E_{1}\right|_{X(\sigma)}} R_{F, \widetilde{F}}\right) \\
& =\operatorname{td}\left(-E_{1}\right)\left(\rho_{*}^{\prime}\left(\operatorname{ch}\left(\mathcal{O}_{D}^{\vee}\right) \operatorname{td}\left(\mathcal{O}_{\tilde{X}}(-D)\right) 0_{\widetilde{E}_{1}^{\prime}}^{!}\left(\tau_{\widetilde{E}_{1}^{\prime}} \widetilde{F}\right)\right)+0_{\left.E_{1}\right|_{X(\sigma)}}^{!}\left(\tau_{\left.E_{1}\right|_{X(\sigma)}} R_{F, \widetilde{F}}\right)\right) \\
& =\operatorname{td}\left(-E_{1}\right)\left(\rho_{*}^{\prime}\left(-D \cdot 0_{\widetilde{E}_{1}^{\prime}}^{!}\left(\tau_{\widetilde{E}_{1}^{\prime}} \widetilde{F}\right)\right)+0_{\left.E_{1}\right|_{X(\sigma)}}^{{ }_{\left(E_{1}\right.}}\left(\tau_{\left.E_{1}\right|_{X(\sigma)}} R_{F, \widetilde{F}}\right)\right) \\
& =\operatorname{td}\left(-E_{1}\right) 0_{E_{1}, \sigma}^{!}\left(\tau_{E_{1}(\sigma)}[F]\right)
\end{aligned}
$$

because $\operatorname{ch}\left(\mathcal{O}_{D}^{\vee}\right) \operatorname{td}\left(\mathcal{O}_{\tilde{X}}(-D)\right)=-D \operatorname{as} \operatorname{ch}\left(\mathcal{O}_{D}^{\vee}\right)=\operatorname{ch}\left[\mathcal{O}_{\tilde{X}} \rightarrow \mathcal{O}_{\tilde{X}}(D)\right]=1-e^{D}$. This proves (5.21) and the theorem.

\section{Application to K-theoretic Landau-Ginzburg/Calabi-Yau CORRESPONDENCE}

In this section, we apply the cosection localized virtual structure sheaf to a K-theoretic Landau-Ginzburg/Calabi-Yau correspondence.

6.1. K-theoretic FJRW invariant. The cosection localized virtual structure sheaf enables us to define the K-theoretic FJRW invariant for narrow sector. To simplify the discussion, we focus on the Fermat quintic case

$$
\sum_{i=1}^{5} z_{i}^{5}: \mathbb{C}^{5} \longrightarrow \mathbb{C}
$$

Let $S$ be the moduli space of 5 -spin curves, i.e. triples $(C, L, p)$ of

(1) a 1-dimensional projective Deligne-Mumford stack $C$ with at worst nodal singularities whose stabilizer groups at nodes or marked points are $\mathbb{Z}_{5}$ which acts on a node $(z w=0)$ as $\zeta \cdot(z, w)=\left(\zeta z, \zeta^{-1} w\right)$ for $\zeta^{5}=1$,

(2) a line bundle $L$ on $C$, and 
(3) an isomorphism $p: L^{5} \rightarrow \omega_{C}^{\log }$.

Assume all the marked points are narrow, i.e. the action of the stabilizer group $\mathbb{Z}_{5}$ on the fiber of $L$ at a marked point is not trivial.

Let $X$ be the Deligne-Mumford stack of quadruples $(C, L, p, x)$ where $(C, L, p) \in S$ and $x \in H^{0}(L)^{\oplus 5}$. By [6, 7], there is a perfect obstruction theory on $X$ and a cosection $\sigma: O b_{X} \rightarrow \mathcal{O}_{X}$ whose zero locus $X(\sigma)$ is the proper Deligne-Mumford stack $S$. As the relative obstruction theory of $X / S$ can be presented by a two-term complex of vector bundles on $X$, we can apply Theorem 5.1 to obtain the cosection localized virtual structure sheaf

$$
\left[\mathcal{O}_{X, \text { loc }}^{\operatorname{vir}}\right] \in K_{0}(S)
$$

To obtain numerical invariants, we take the Euler characteristic.

Definition 6.1. The K-theoretic Fan-Jarvis-Ruan-Witten invariant is defined by

$$
\chi_{\mathrm{loc}}^{\mathrm{vir}}(X, \beta)=\chi\left(S, \mathcal{O}_{X, \mathrm{loc}}^{\mathrm{vir}} \cdot \beta\right), \quad \beta \in K^{0}(S) .
$$

Since $S$ is a smooth Deligne-Mumford stack, the Kawasaki-Riemann-Roch theorem enables us to express the numerical K-theoretic FJRW invariant as (cosection localized) virtual integrals.

In 9], based on the Polishchuk-Vaintrob construction of Witten's top Chern class 34, Chiodo constructed a K-theory class $K e\left(E^{\vee}, \tau^{\vee}\right) \in K_{0}(S)$ whose top Chern class

$$
c_{\text {top }}(E, \tau)=\operatorname{ch}\left(K e\left(E^{\vee}, \tau^{\vee}\right)\right) \operatorname{td}(E)^{-1} \in A_{*}(S)
$$

coincides with the cosection localized virtual fundamental class $[X]_{\mathrm{loc}}^{\mathrm{vir}}$ in $A_{*}(S)$ by [7, §5.2] and [9, Theorem 5.4.1]. We have the following comparison result.

Proposition 6.2. Chiodo's K-theory class $K e\left(E^{\vee}, \tau^{\vee}\right)$ coincides with the cosection localized virtual structure sheaf $\left[\mathcal{O}_{X, \mathrm{loc}}^{\mathrm{vir}}\right]$ in $K_{0}(S)$.

Proof. The same argument as in the proof of [7, Proposition 5.10] also proves this proposition. Indeed, since the pushforward and the localized Gysin map

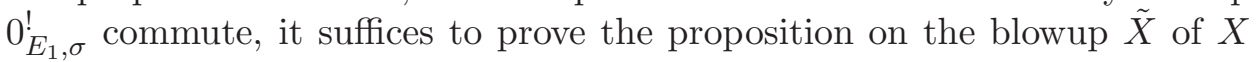
along $X(\sigma)$ where we have an exact sequence

$$
0 \longrightarrow \widetilde{E}_{1}^{\prime} \longrightarrow \widetilde{E}_{1} \longrightarrow \mathcal{O}_{\tilde{X}}(-D) \longrightarrow 0 .
$$

Here $D$ denotes the exceptional divisor. By deforming this exact sequence to the split case $\widetilde{E}_{1}=\widetilde{E}_{1}^{\prime} \oplus \mathcal{O}_{\tilde{X}}(-D)$, the proposition is reduced to the case of vanishing cosection $\widetilde{E}_{1}^{\prime} \rightarrow 0$ and the case where cosection is the natural inclusion $\mathcal{O}_{\tilde{X}}(-D) \rightarrow \mathcal{O}_{\tilde{X}}$. Each of these cases is easy to check. 
6.2. GSW model for K-theoretic Gromov-Witten invariant. Consider the Fermat quintic Calabi-Yau 3-fold

$$
Y=\left(\sum_{i=1}^{5} z_{i}^{5}=0\right) \subset \mathbb{P}^{4} .
$$

Let $Z$ be the deformation of $\mathbb{P}^{4}$ to the normal cone $\mathcal{O}_{Y}(5)$ of $Y$ in $\mathbb{P}^{4}$, i.e. $Z$ is the complement of the proper transform of $\{0\} \times \mathbb{P}^{4}$ in the blowup of $\mathbb{P}^{1} \times \mathbb{P}^{4}$ along $\{0\} \times Y$. Let

$$
p: Z \longrightarrow \mathbb{P}^{1} \times \mathbb{P}^{4} \longrightarrow \mathbb{P}^{1}
$$

denote the composition. Then $Z_{t}=t \times_{\mathbb{P}^{1}} Z$ is $\mathbb{P}^{4}$ for $t \neq 0$ and $\mathcal{O}_{Y}(5)$ for $t=0$.

Let $N=\bar{M}_{g}(Y, d)$ (resp. $\left.M=\bar{M}_{g}\left(\mathbb{P}^{4}, d\right)\right)$ be the moduli space of stable maps to $Y$ (resp. $\left.\mathbb{P}^{4}\right)$. Let $M^{p}=\bar{M}_{g}\left(\mathbb{P}^{4}, d\right)^{p}$ denote the moduli space of pairs $(f, p)$ where $\left(f: C \rightarrow \mathbb{P}^{4}\right) \in M$ and $p \in H^{0}\left(f^{*} \mathcal{O}_{\mathbb{P}^{4}}(-5) \otimes \omega_{C}\right)$.

Let $\bar{X}=\bar{M}_{g}\left(Z / \mathbb{P}^{1}, d\right)$ be the moduli space of stable maps of genus $g$ and degree $d$ to the fibers of $p$. Let

$$
\pi: X=\bar{M}_{g}\left(Z / \mathbb{P}^{1}, d\right)^{p} \longrightarrow \mathbb{P}^{1}
$$

denote the moduli space of pairs $(f, p)$ where $(f: C \rightarrow Z) \in \bar{X}$ and $p \in$ $H^{0}\left(f^{*} \mathcal{O}_{\mathbb{P}^{4}}(-5) \otimes \omega_{C}\right)(\mathrm{cf} .[6, \S 4.1])$. It is straightforward that $X_{t}$ is $M^{p}$ for $t \neq 0$ and the central fiber $X_{0}$ is the moduli space of triples $(f, s, p)$ where $(f$ : $C \rightarrow Y) \in N=\bar{M}_{g}(Y, d), s \in H^{0}\left(f^{*} \mathcal{O}_{Y}(5)\right)$ and $p \in H^{0}\left(f^{*} \mathcal{O}_{Y}(-5) \otimes \omega_{C}\right)$. By [6, §4], all the assumptions of Proposition 5.5 are satisfied and hence we

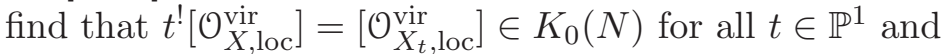

$$
\chi\left(N, \mathcal{O}_{M^{p}, \text { loc }}^{\mathrm{vir}}\right)=\chi\left(N, \mathcal{O}_{X_{0}, \text { loc }}^{\mathrm{vir}}\right) .
$$

Here $X(\sigma)=N \times \mathbb{P}^{1}$. On the other hand, the proof of [6, Theorem 5.7] together with Example 4.2 above proves

$$
\chi\left(N, \mathcal{O}_{X_{0}, \text { loc }}^{\mathrm{vir}}\right)=(-1)^{5 d-g+1} \chi\left(N, \mathcal{O}_{N}^{\mathrm{vir}}\right)=(-1)^{5 d-g+1} \chi^{\mathrm{vir}}(N) .
$$

We therefore proved the following.

Proposition 6.3. The cosection localized virtual Euler characteristic

$$
\chi_{\mathrm{loc}}^{\operatorname{vir}}\left(\bar{M}_{g}\left(\mathbb{P}^{4}, d\right)^{p}\right):=\chi\left(N, \mathcal{O}_{M^{p}, \text { loc }}^{\mathrm{vir}}\right)
$$

of $M^{p}$ is equal to the K-theoretic Gromov-Witten invariant $\chi\left(N, \mathcal{O}_{N}^{\text {vir }}\right)$ of $N$ up to sign by

$$
\chi_{\operatorname{loc}}^{\operatorname{vir}}\left(\bar{M}_{g}\left(\mathbb{P}^{4}, d\right)^{p}\right)=(-1)^{5 d-g+1} \chi^{\operatorname{vir}}(N) .
$$

The Landau-Ginzburg/Calabi-Yau correspondence predicts that the FJRW invariant of the Landau-Ginzburg model (6.1) is equivalent to the GW invariant of the Calabi-Yau 3-fold $Y$ via variable changes, analytic continuations, and symplectic transformations. Proposition 6.3 may be useful for the K-theoretic LG/CY correspondence. 


\section{REFERENCES}

1. K. Behrend. Donaldson-Thomas type invariants via microlocal geometry. Ann. of Math. (2) 170 (2009), no. 3, 1307-1338.

2. K. Behrend and B. Fantechi. The intrinsic normal cone. Invent. Math. 128 (1997), no. $1,45-88$.

3. A. Borel and J-P. Serre. Le thorme de Riemann-Roch. Bull. Soc. Math. France 86 (1958) $97-136$.

4. H.-L. Chang and Y.-H. Kiem. Poincaré invariants are Seiberg-Witten invariants. Geom. Topol. 17 (2013), no. 2, 1149-1163.

5. H.-L. Chang, Y.-H. Kiem and J. Li. Torus localization and wall crossing for cosection localized virtual cycles. Adv. Math. 308 (2017), 964-986.

6. H.-L. Chang and J. Li. Gromov-Witten invariants of stable maps with fields. Int. Math. Res. Not. IMRN 2012, no. 18, 4163-4217.

7. H.-L. Chang, J. Li and W.-P. Li. Witten's top Chern class via cosection localization. Invent. Math. 200 (2015), no. 3, 1015-1063.

8. H.-L. Chang, J. Li, W.-P. Li and M. C.-C. Liu. Mixed-Spin-P fields of Fermat quintic polynomials. Preprint, arXiv:1505.07532

9. A. Chiodo. The Witten top Chern class via K-theory. Jour. Alg. Geom. 15 (2006) 681-707.

10. E. Clader. Landau-Ginzburg/Calabi-Yau correspondence for the complete intersections $X_{3,3}$ and $X_{2,2,2,2}$. Adv. Math. 307 (2017), 1-52.

11. H. Fan, T. Jarvis and Y. Ruan. The Witten equation, mirror symmetry, and quantum singularity theory. Ann. of Math. (2) 178 (2013), no. 1, 1-106.

12. B. Fantechi and L. Göttsche. Riemann-Roch theorems and elliptic genus for virtually smooth schemes. Geom. Topol. 14 (2010), no. 1, 83-115.

13. W. Fulton. Intersection theory. Ergebnisse der Mathematik und ihrer Grenzgebiete. 3. Folge. 2. Springer-Verlag, Berlin, 1998.

14. A. Gholampour and A. Sheshmani. Donaldson-Thomas Invariants of 2-Dimensional sheaves inside threefolds and modular forms. Preprint, arXiv:1309.0050.

15. A. Gholampour and A. Sheshmani. Invariants of pure 2-dimensional sheaves inside threefolds and modular forms. Preprint, arXiv:1305.1334.

16. T. Graber and R. Pandharipande. Localization of virtual cycles. Invent. Math. 135 (1999), no. 2, 487-518.

17. J. Hu, W.-P. Li, Z. Qin. The Gromov-Witten invariants of the Hilbert schemes of points on surfaces with $p_{g}>0$. Internat. J. Math.26 (2015), no. 1, 1550009, 26 pp.

18. Y. Jiang and R. Thomas. Virtual signed Euler characteristics. J. Algebraic Geom. 26 (2017), no. 2, 379-397.

19. Y.-H. Kiem and J. Li. Localizing virtual cycles by cosections. J. Amer. Math. Soc. 26 (2013), no. 4, 1025-1050.

20. Y.-H. Kiem and J. Li. Categorification of Donaldson-Thomas invariant via perverse sheaves. Preprint, arXiv: 1212.6444.

21. Y.-H. Kiem and J. Li. Low degree $G W$ invariants of spin surfaces. Pure Appl. Math. Q. 7 (2011), no. 4, Special Issue: In memory of Eckart Viehweg, 1449-1475.

22. Y.-H. Kiem and J. Li. Low degree GW invariants of surfaces II. Sci. China Math. 54 (2011), no. 8, 1679-1706.

23. Y.-H. Kiem and J. Li. A wall crossing formula of Donaldson-Thomas invariants without Chern-Simons functional. Asian J. Math. 17 (2013), no. 1, 63-94.

24. B. Kim, A. Kresch and T. Pantev. Functoriality in intersection theory and a conjecture of Cox, Katz, and Lee. J. Pure Appl. Algebra 179 (2003), no. 1-2, 127-136.

25. S. Kleiman Intersection theory and enumerative geometry: a decade in review. With the collaboration of Anders Thorup on 3. Proc. Sympos. Pure Math., 46, Part 2, 
Algebraic geometry, Bowdoin, 1985 (Brunswick, Maine, 1985), 321-370, Amer. Math. Soc., Providence, RI, 1987.

26. M. Kool and R. Thomas. Reduced classes and curve counting on surfaces I: theory. Algebr. Geom. 1 (2014), no. 3, 334-383.

27. A. Kresch. Cycle groups for Artin stacks. Invent. Math. 138 (1999), no. 3, 495-536.

28. S. Lang. Algebra. Revised third edition. Graduate Texts in Mathematics, 211. Springer-Verlag, New York, 2002. xvi+914 pp.

29. Y.P. Lee. Quantum K-theory. I. Foundations. Duke Math. J. 121 (2004), no. 3, 389424.

30. J. Li and G. Tian. Virtual moduli cycles and Gromov-Witten invariants of algebraic varieties. J. Amer. Math. Soc. 11 (1998), no. 1, 119-174.

31. Y. Manin. Lectures in algebraic geometry. Part II: The K-functor in algebraic geometry. Izdat. Moskov. Univ., Moscow, 1971. 86 pp.

32. D. Maulik, R. Pandharipande and R. Thomas. Curves on K3 surfaces and modular forms. With an appendix by A. Pixton. J. Topol. 3 (2010), no. 4, 937-996.

33. R. Pandharipande and R Thomas. The Katz-Klemm-Vafa conjecture for K3 surfaces. Forum Math. Pi 4 (2016), e4, 111 pp.

34. A. Polishchuk and A. Vaintrob. Algebraic construction of Witten's top Chern class. Contemporary Mathematics, vol. 276, pp. 229?249. American Mathematical Society, Providence (2001)

35. J. Shen. Cobordism invariants of the moduli space of stable pairs. J. Lond. Math. Soc. (2) 94 (2016), no. 2, 427-446.

36. B. Totaro. The resolution property for schemes and stacks. J. Reine Angew. Math. $\mathbf{5 7 7}$ (2004), 1-22.

37. A. Vistoli. Intersection theory on algebraic stacks and on their moduli spaces. Invent. Math. 97 (1989), no. 3, 613-670.

Department of Mathematics and Research Institute of Mathematics, Seoul National University, Seoul 08826, Korea

E-mail address: kiem@snu.ac.kr

Department of Mathematics, Stanford University, CA 94305, USA

E-mail address: jli@math.stanford.edu 